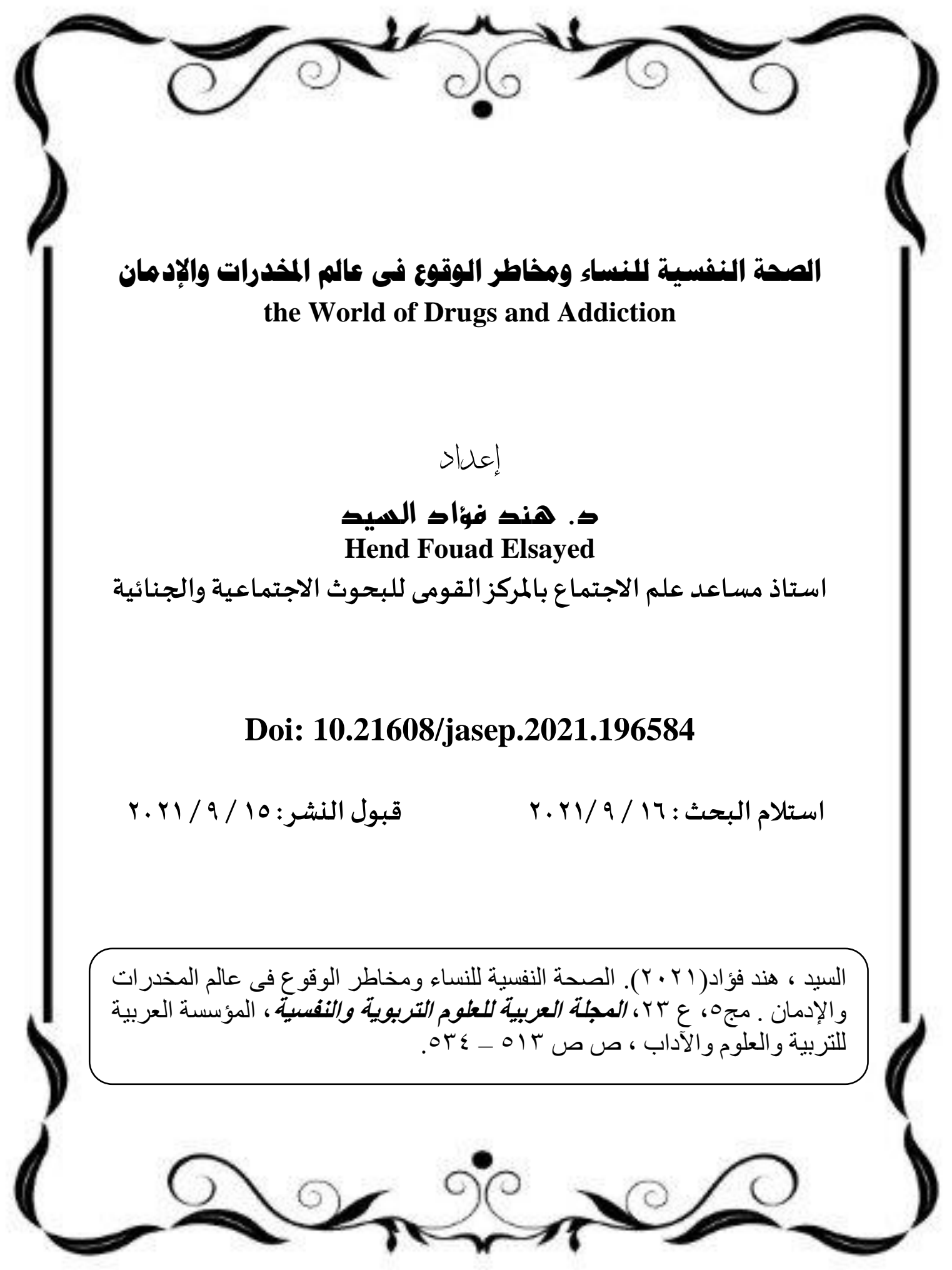




\section{الصحة النفسية للنساء ومخاطر الوقوع فى عالم المخدرات والإدمان}

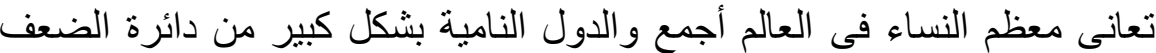
المركبة تجاه نو عها، فهذا الضعف المركب يجعلها فريسة للإصابة بالاضطر ابات النفسية فى مئي كثير من الأحيان نتيجة للضغوط الاجتماعية و الاقتصادية والأسرية التى تعانى منها طيلة

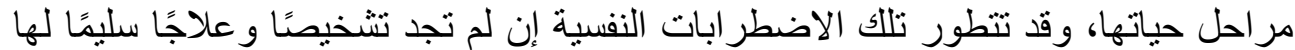

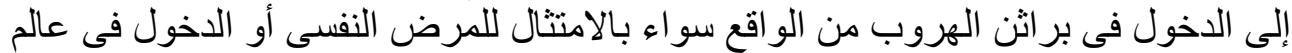
المخدرات والإدمان، ورنا تعانى من حمل مضاعف حينما تدمّن المخدرات؛ حيث تتحمل

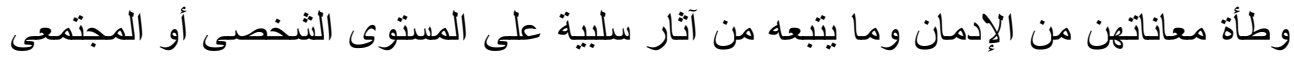

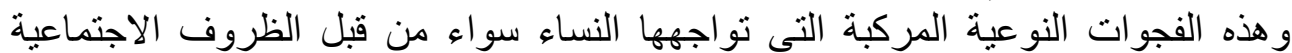
و الاقتصادية والأسرية والتى تعرضنهن لمخاطر الاضطر ابات النفسية أو قد تؤدى بهن إلى فئى الإدمان تجعلهن تعانين من فجوة نوعية ضدهن فى رحلتهن مع الإدمان سواء من حيث

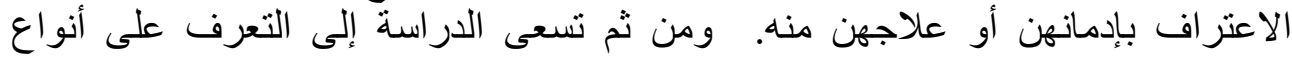

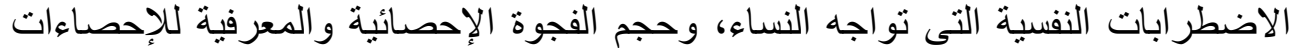

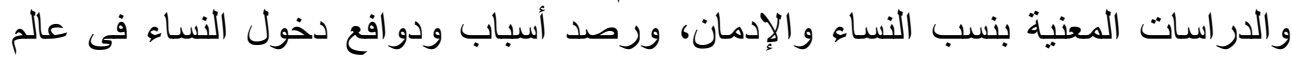

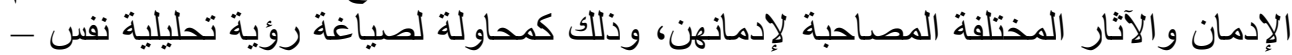

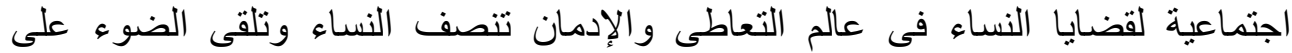

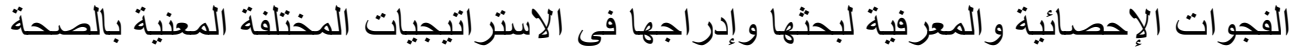

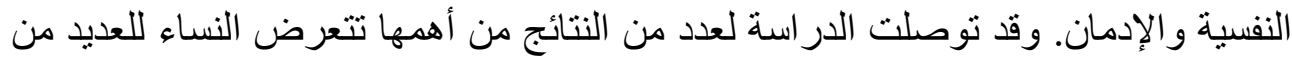
الأمر اض النفسية والاضطر ابات فى شخصيتهن بسبب العو امل الاجتماعية والأسرية و البيئية

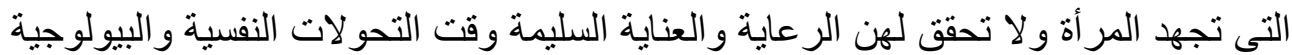

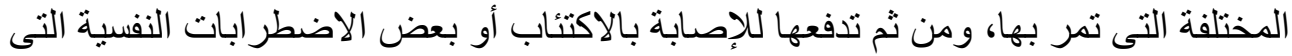
تختلف قوتها و التكيف معها وفقا لكل حالة. وكثفت الدراسة عن وجود فجوة معرفية

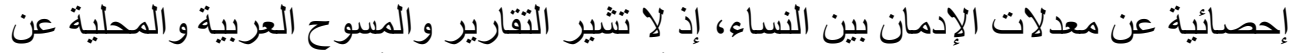
حجم مشكلة التعاطى و الإدمان بين النساء بالأرقام الفعلية وذللك لأسباب متعددة منها العاديات العادات و التقاليد وسيطرتها على بعض العائلات، بجانب سيادة الثقافة الذكورية التى لا تسمح

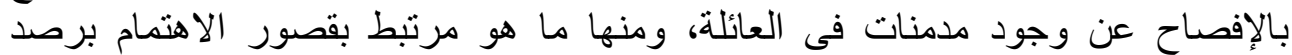

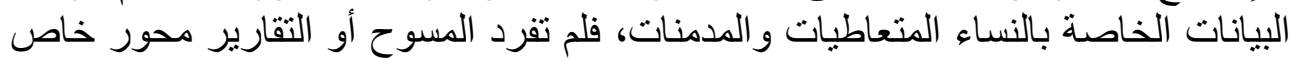

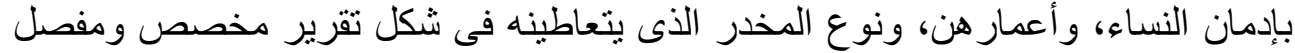

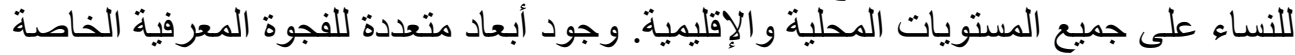

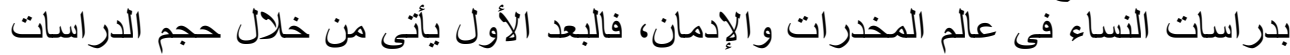

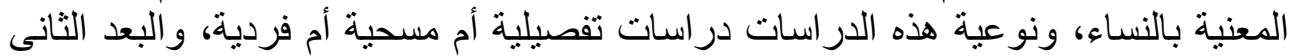




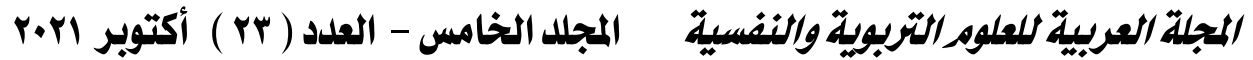

يتمثل فى حجم العينات التى تم دراستها فى هذه الدراسات، و غلبة الدراسات الفردية ذات التهات

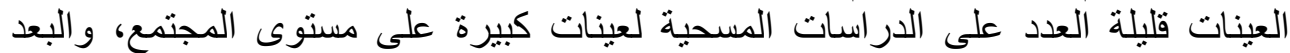

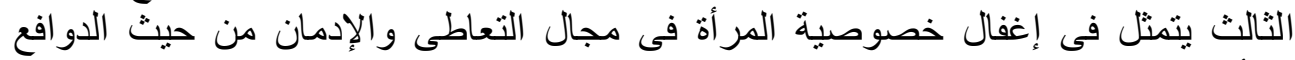

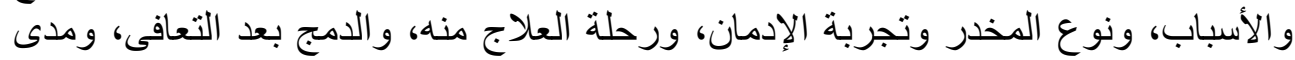

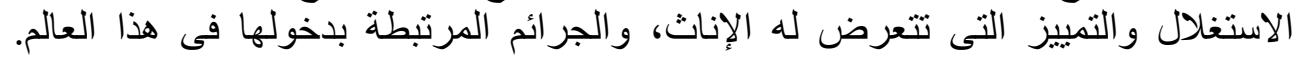

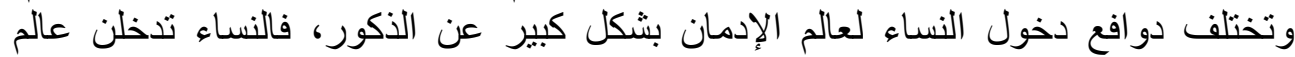

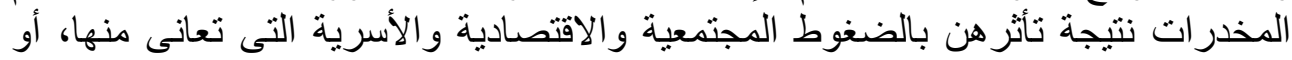

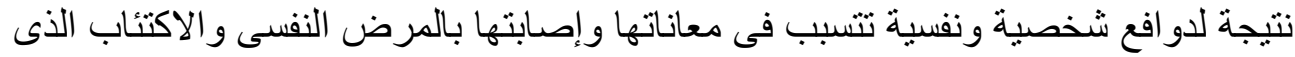
قد يدفعهن للهروب من الالآم و الو اقع و الانسياق في عانئ والم المخدرات والإدمان.

\section{Abstract:}

Most women in the whole world and developing countries suffer greatly from the accumulated cycle of vulnerability towards their gender This accumulated weakness makes her prey to mental disorders in many cases as a result of the social, economic and family pressures she suffers from throughout her life stages. If these mental disorders do not find a proper diagnosis and treatment, they may develop into an escape from reality, whether by complying with mental illness or entering the world of drugs and addiction, Where they bear the brunt of their suffering from addiction and the negative effects that follow at the personal or societal level. These accumulated qualitative gaps that women face, whether from social, economic and family conditions, which expose them to the risks of psychological disorders or may lead them to addiction, make them suffer from a qualitative gap against them in their journey with Addiction, whether in terms of recognizing or treating their addiction. This study seeks to identify the types of psychological disorders facing women, the size of the statistical and knowledge gap for statistics and studies concerned with the percentage of women and addiction, and to monitor the reasons and motives for women's entry into the world of addiction and the various effects associated with their addiction, as an attempt to formulate a psycho-social analytical vision for women's issues in the world Abuse and addiction do justice to women and highlight 
statistical and knowledge gaps for their investigation and inclusion in the various strategies concerned with mental health and addiction.This study reached a number of results, the most important of which are:Women are exposed to many psychological diseases and disorders in their personality due to social, family and environmental factors that stress women and do not provide them with proper care and care at the time of the various psychological and biological transformations they are going through, and then push them to develop depression or some psychological disorders that differ in strength and adaptation according to each case. The study revealed a statistical knowledge gap about addiction rates among women, as Arab and local reports and surveys do not indicate the extent of the problem of abuse and addiction among women in actual numbers, for various reasons, including customs and traditions and their control over some families, in addition to the dominance of the male culture that does not allow disclosure of The presence of female addicts in the family, some of which is related to the lack of interest in monitoring data on women abusers and addicts. Surveys or reports did not single out a focus on women's addiction, their ages, and the type of drug they use in the form of a dedicated and detailed report for women at all local and regional levels. There are multiple dimensions of the knowledge gap related to women's studies in the world of drugs and addiction, The first dimension comes through the volume of studies concerned with women, and the quality of these studies, detailed, survey or individual studies. The second dimension is represented in the size of the samples that have been studied in these studies, and the predominance of individual studies. With few samples on survey studies of large samples at the community level, and the third dimension is the neglect of the privacy of women in the field of abuse and addiction in terms of motives and causes, type of drug and addiction experience, treatment journey from it, integration after recovery, and the extent of exploitation and discrimination that females are exposed to, And the 


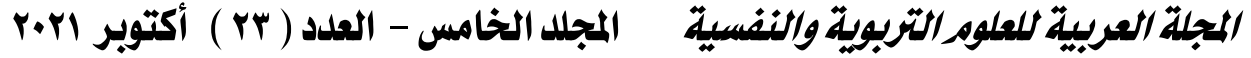

crimes associated with entering this world. Women's motives for entering the world of addiction differ greatly from males, as women enter the world of drugs as a result of being affected by societal, economic and family pressures that they suffer from, or as a result of personal and psychological motives that cause their suffering, mental illness and depression, which may push them to escape from pain, reality and drift in the world of drugs and addiction.

Key Words:Gender, Drugs, Addiction, accumulated Cycle of Vulnerability, Statistical knowledge Gap, Lack of Studies .

مقدمة

على الرغم من أن نسب الإصابة بالاضطرابات النفسية تكاد تتساوى بين النساء

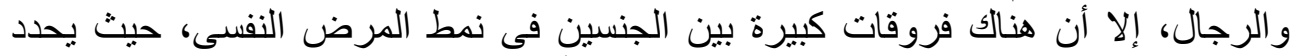

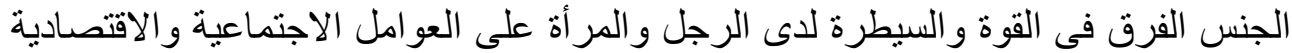

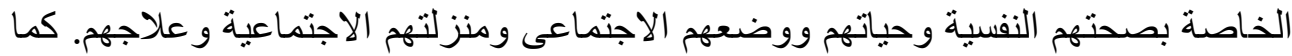

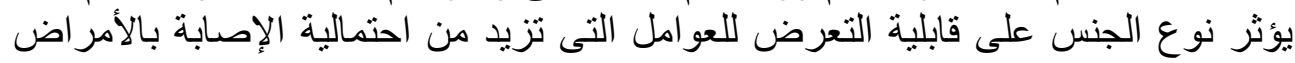

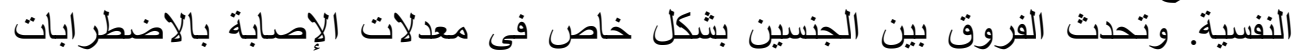

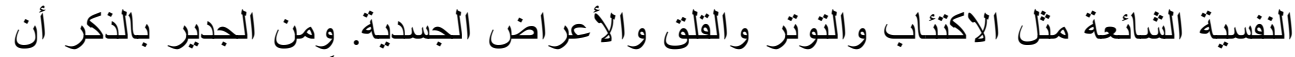

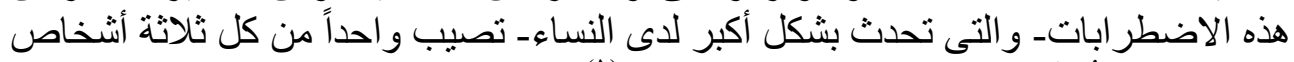

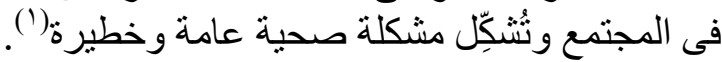

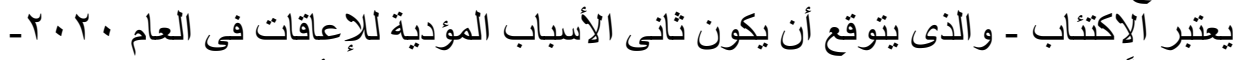

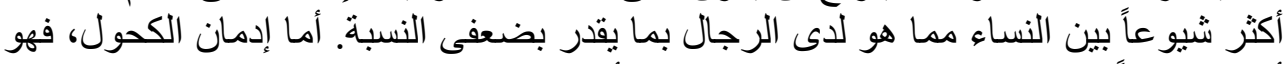

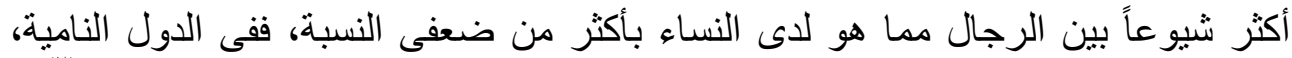

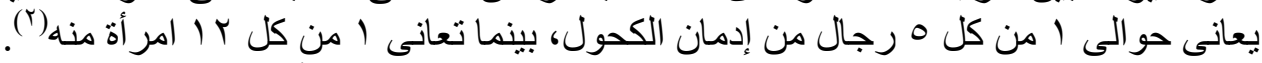

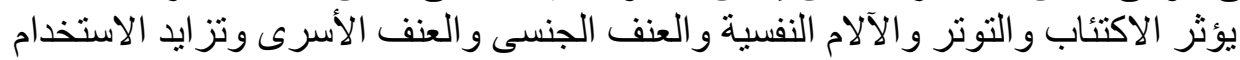

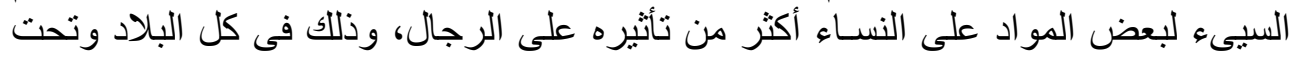

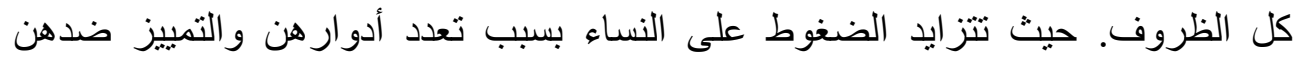

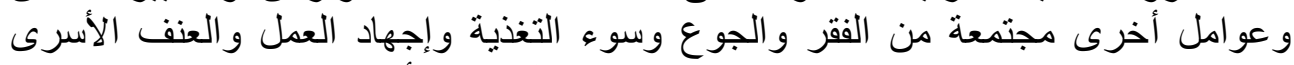

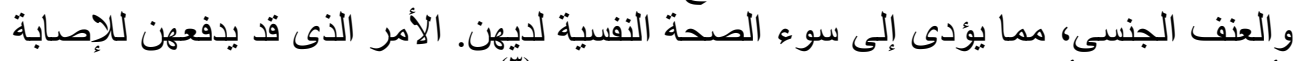

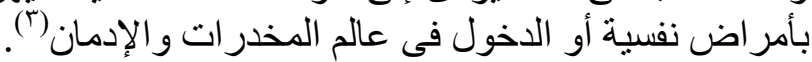

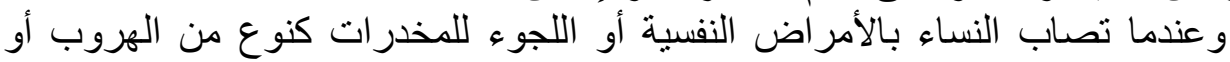

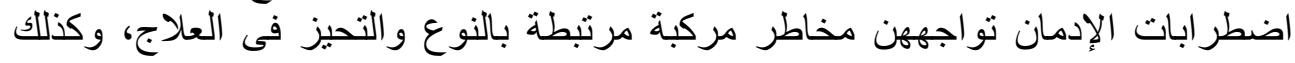




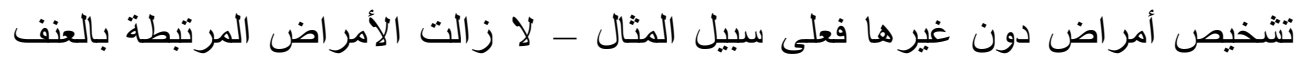

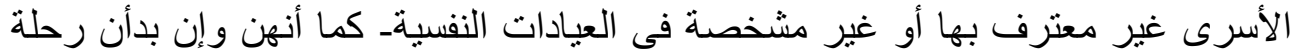

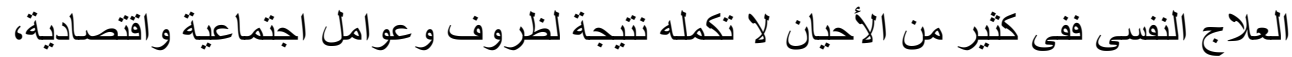

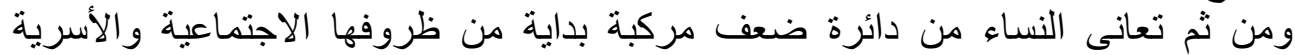

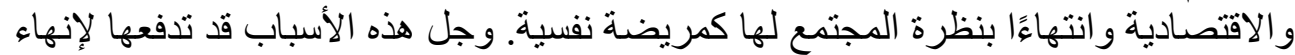

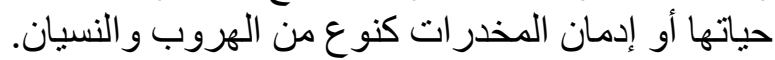

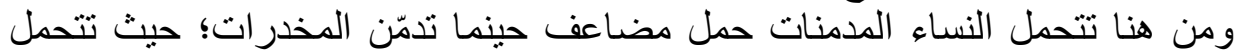

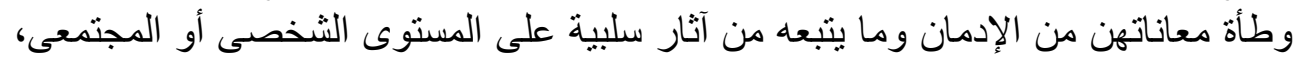

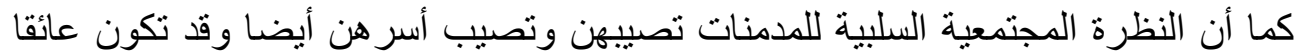

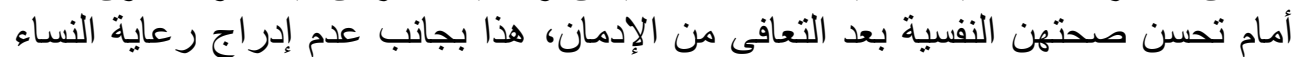

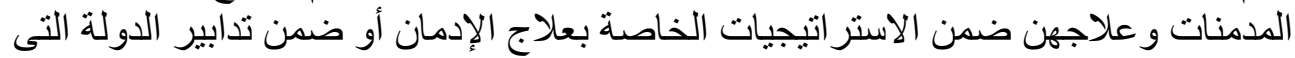

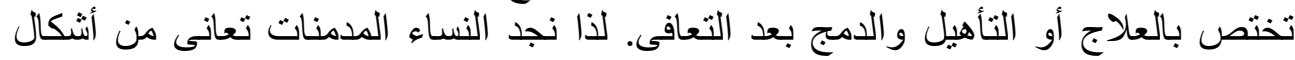

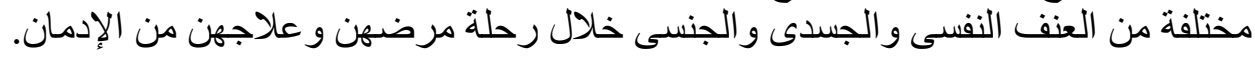

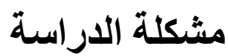
تعانى معظم النساء فى العالم أجمع و الدول النامية بشكل كبير من دائرة الضعف الضعابة المركبة تجاه

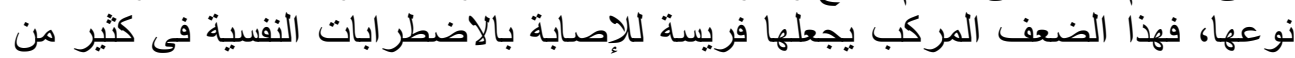

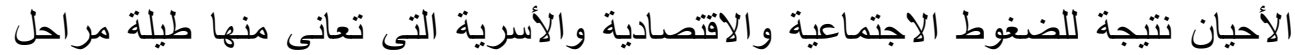

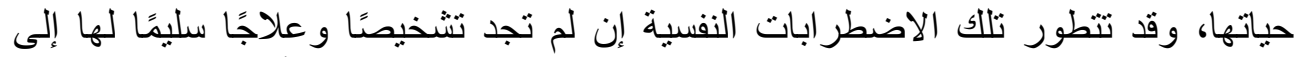

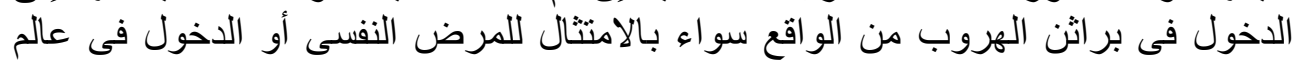

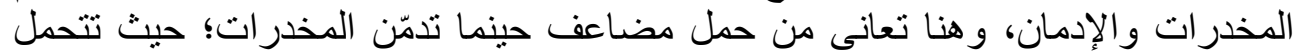

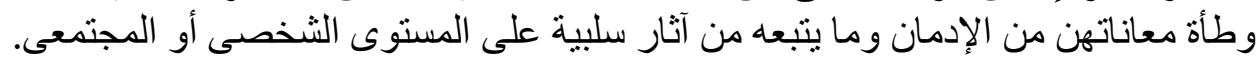

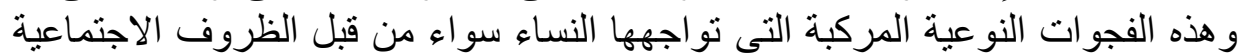

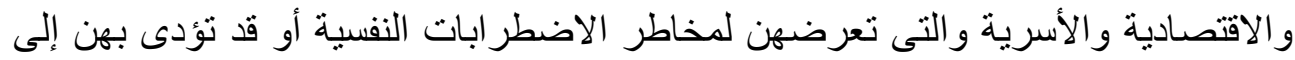

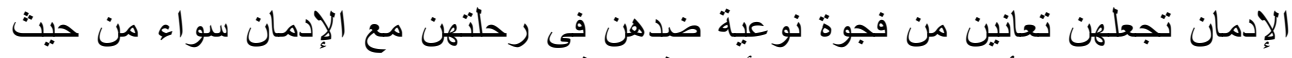

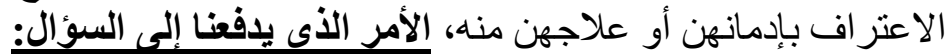

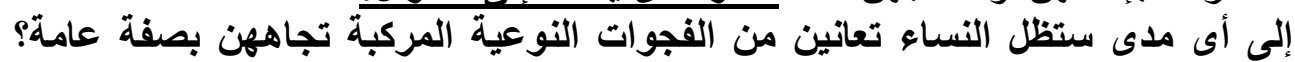

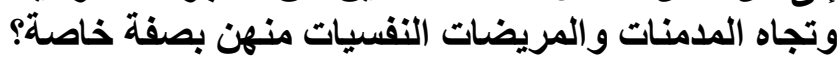
تساؤلات الار اسنة فى سياق الإشكالية التى أوضحناها الإنا تسعى الدر اسة للإجابة على التساؤلات الات التالية:

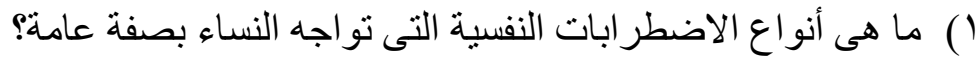

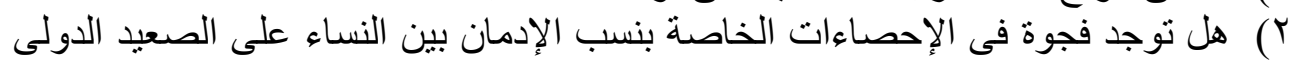
و المحلى؟ 


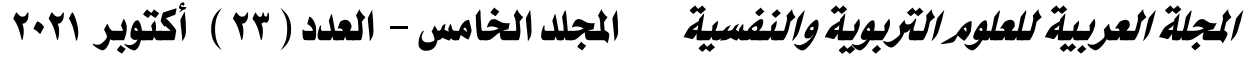

ץ) ما هى أبعاد الفجوة المعرفية للاراسات المعنية بالنساء وعلاقتها بعالم المخدرات

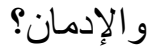

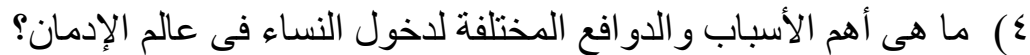

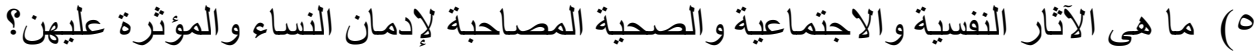

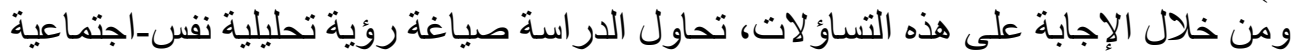

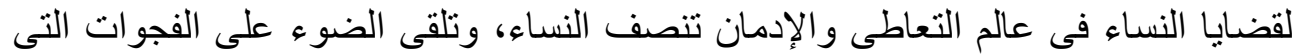
تعانى منها لبحثها و إدر اجها فى الاستر اتيجيات المختلفة المعنية بالصحة الصنان النفسية و الإدمان. منهج الدراسة منها لبحته تتنمى الدر اسة للمنهج الوصفى، المعتمد على استقر اء وتحليل الإحصاءاءت والبه البيانات المختلفة

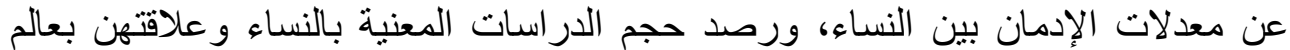

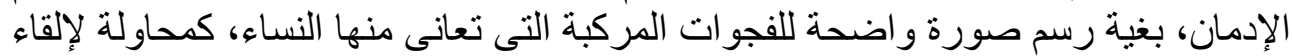

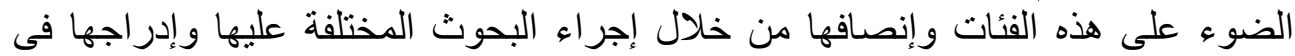

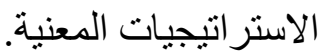

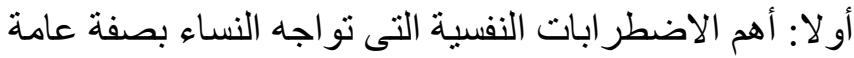

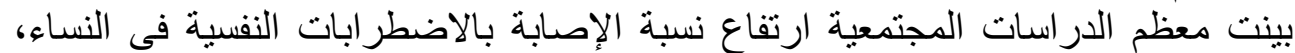

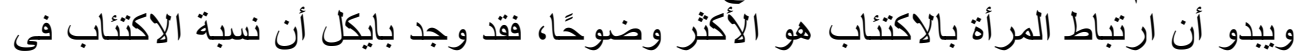

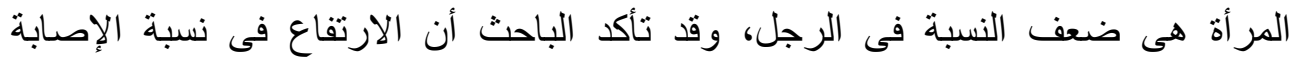

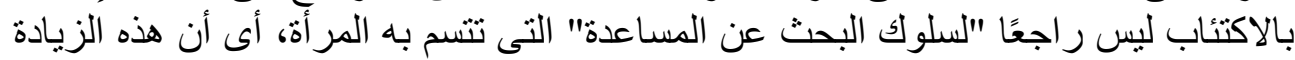

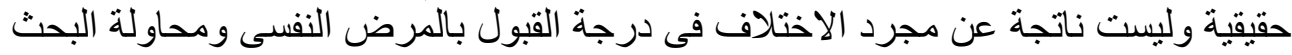

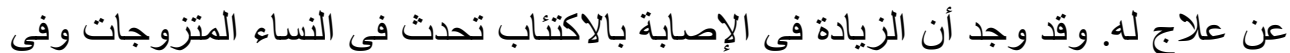

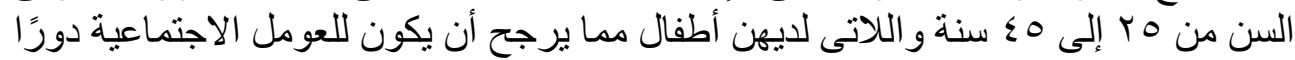

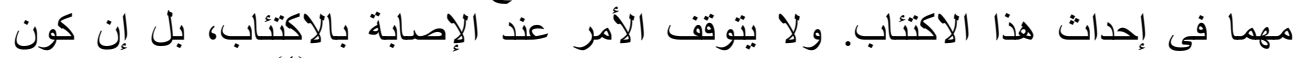

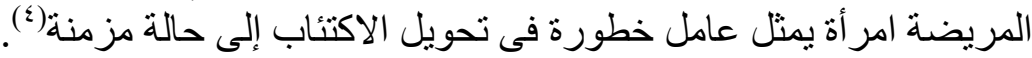

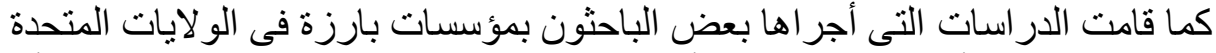

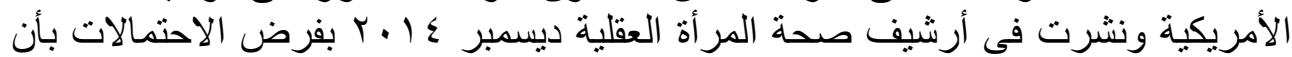

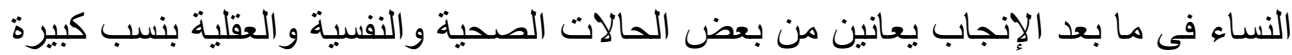

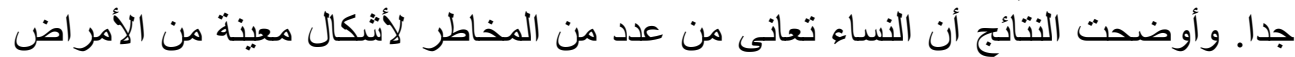

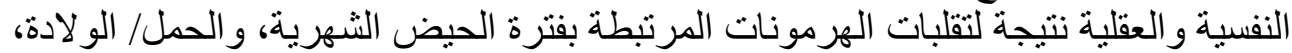

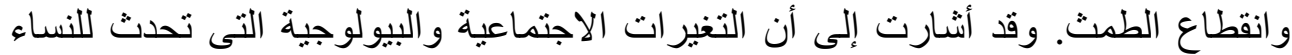

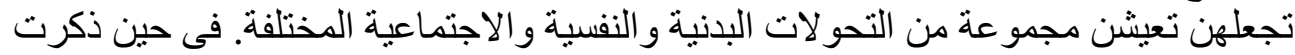

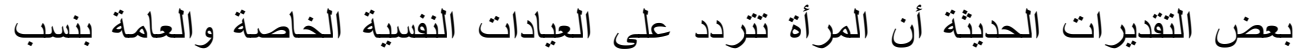

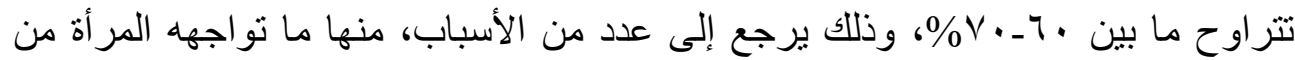


تحولات فى مرحلة منتصف العمر. أو ما تواجها من اضطرابات نفسية أثناء مرحلة إنهاء

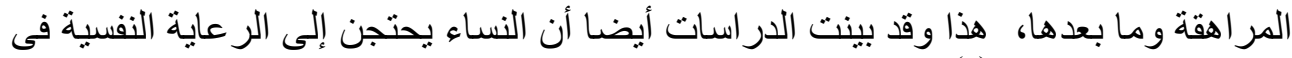
شتى المر احل العمرية).

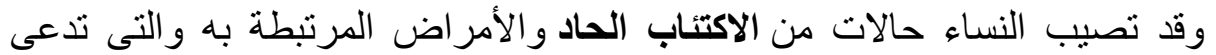

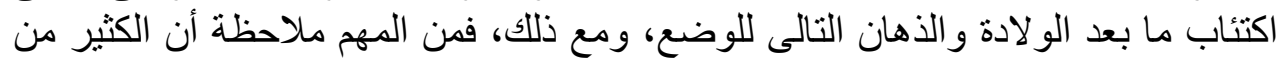

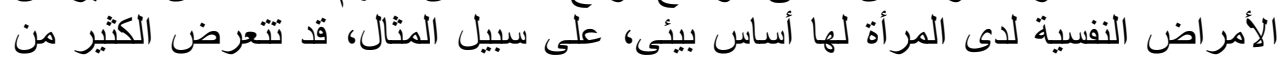

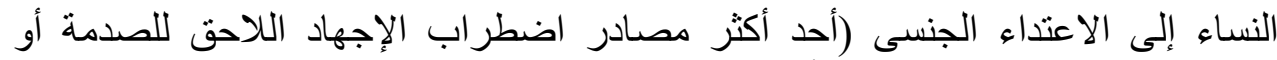

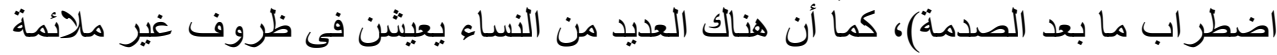

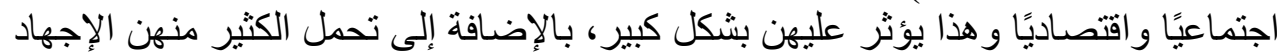

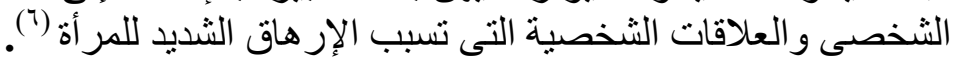

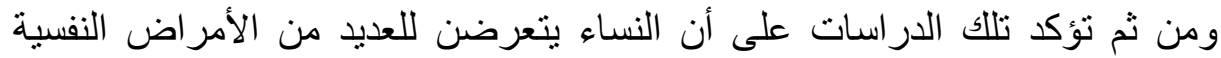

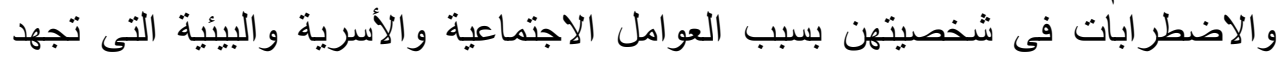

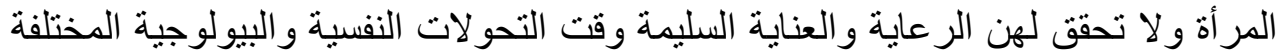

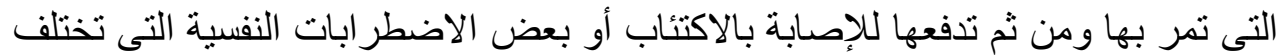

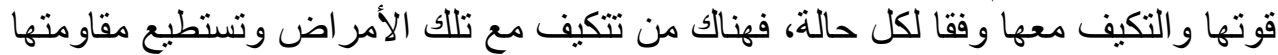

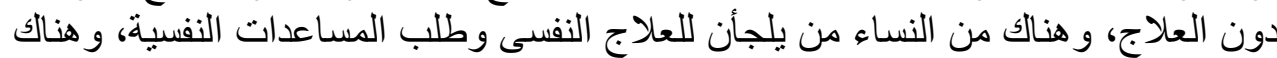

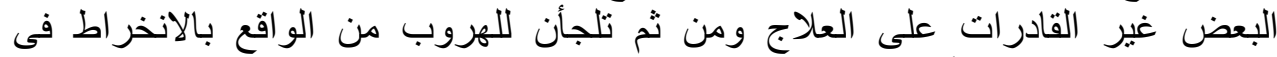

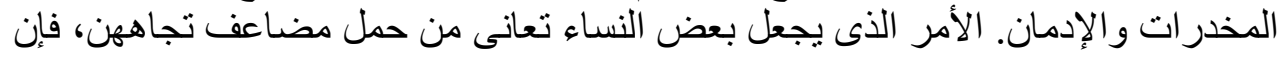

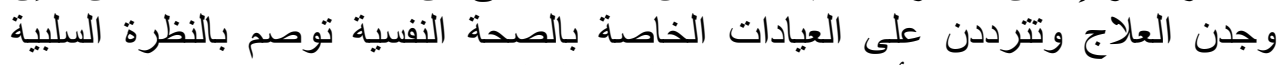

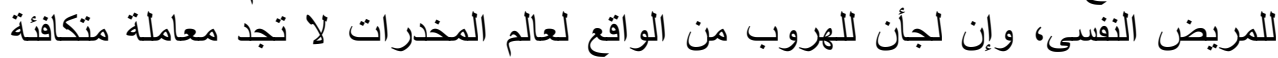

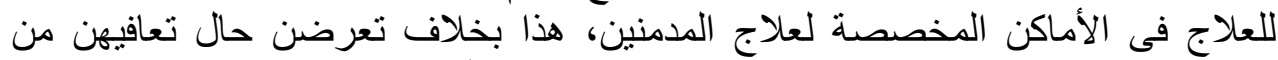

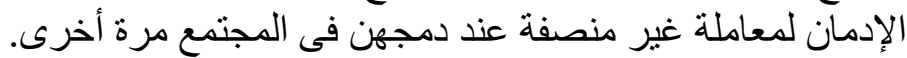

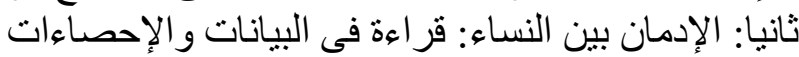

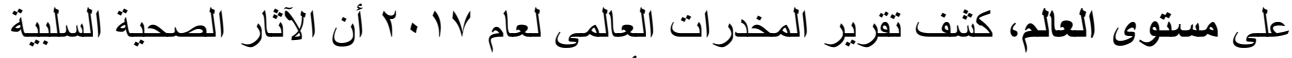

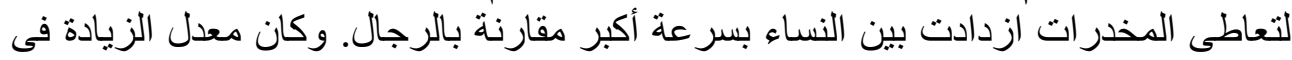

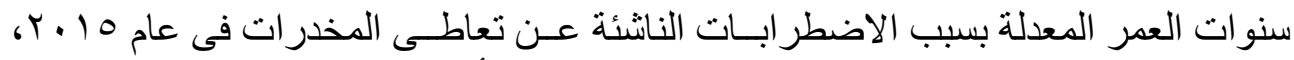

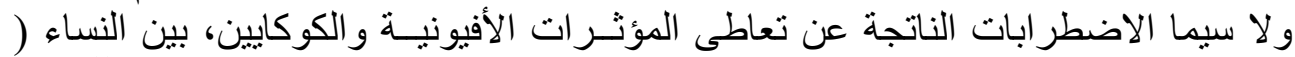

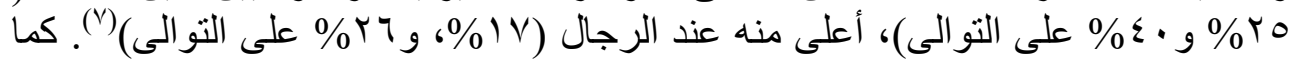

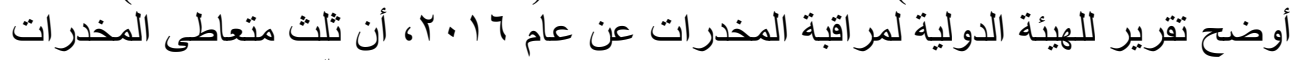

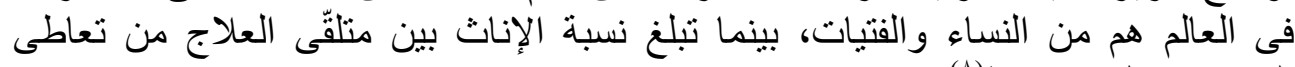

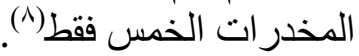




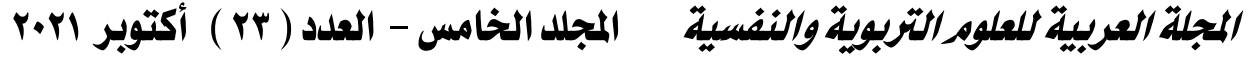

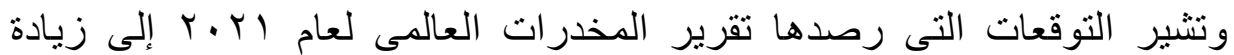

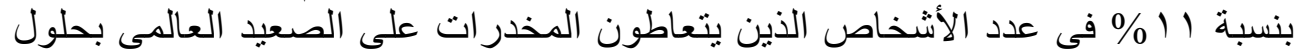

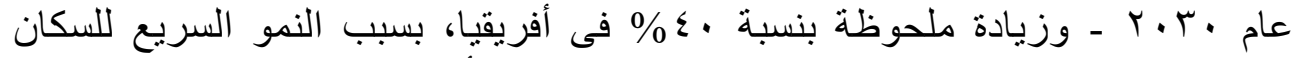

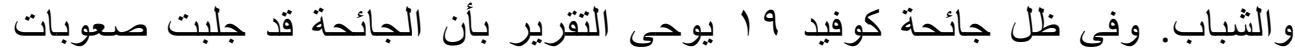

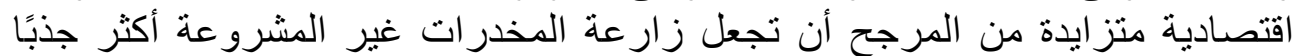

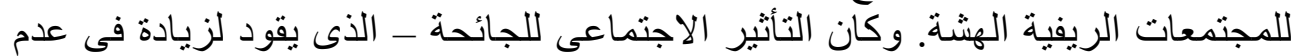

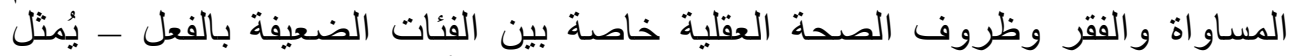

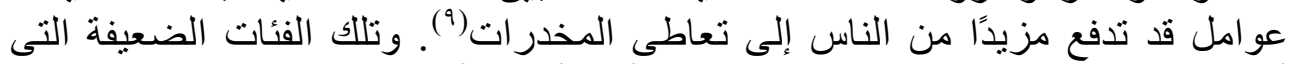

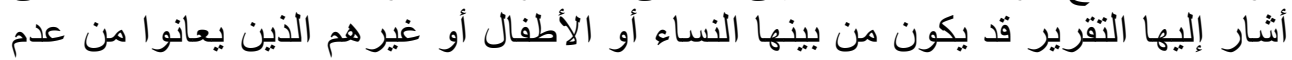

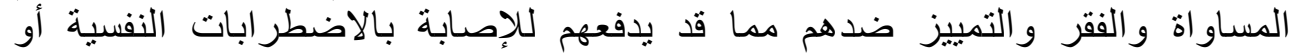

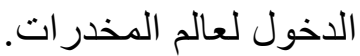

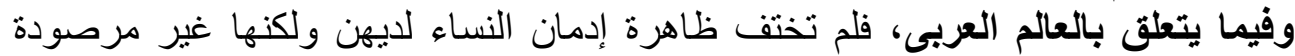

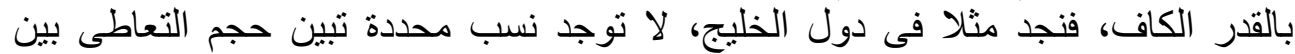

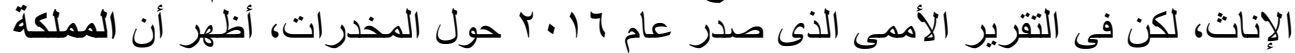

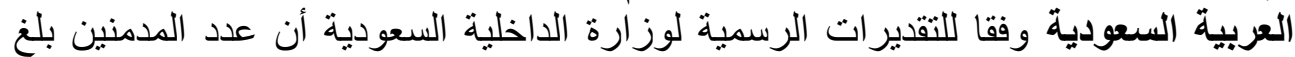

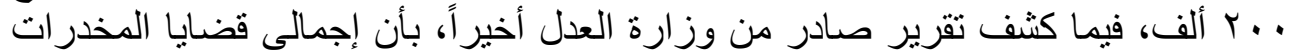

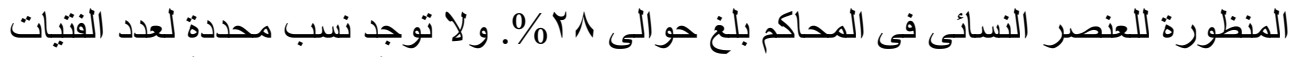

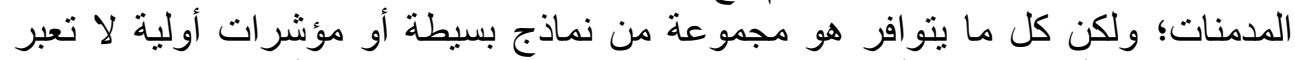

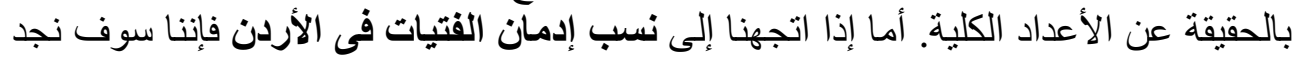

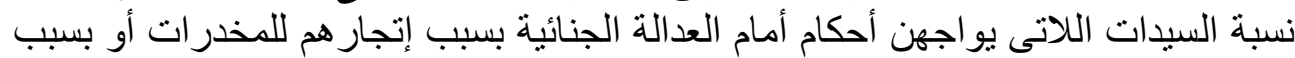

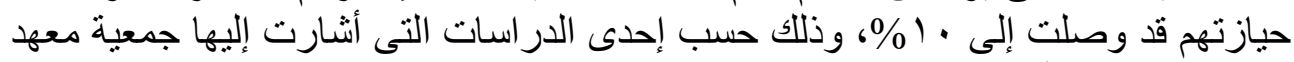

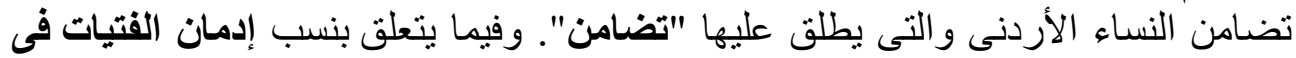

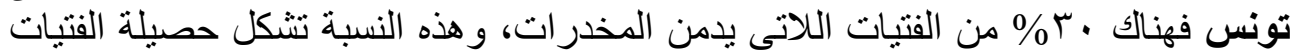

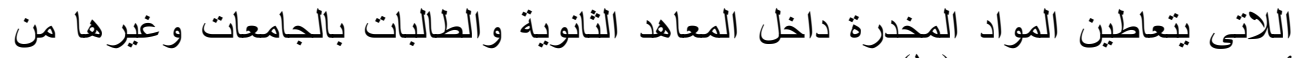

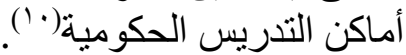

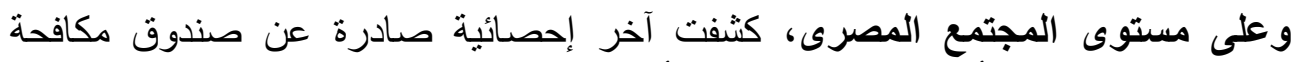

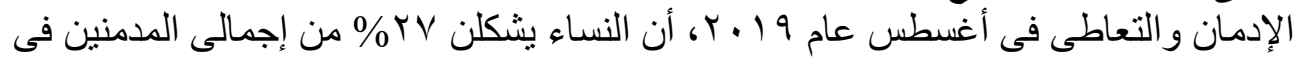

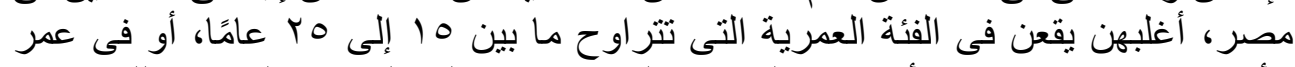

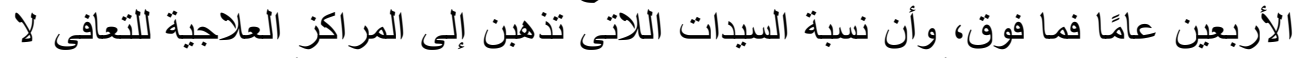

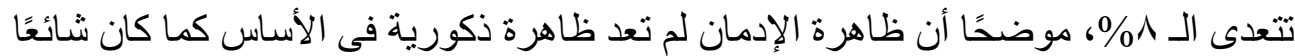

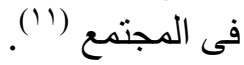


و أثشارت نتائج المسح القومى الثنامل لظاهرة تعاطى و إدمان المواد المؤثرة في الحالة

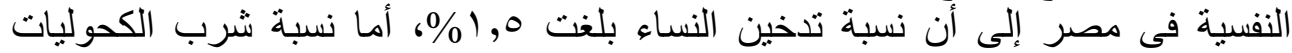

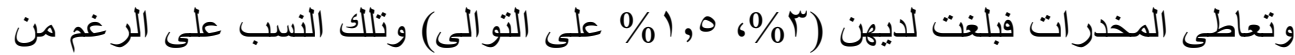

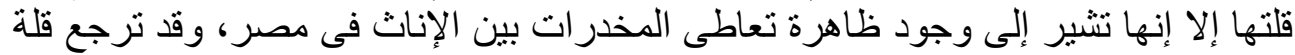

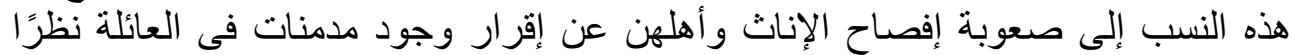

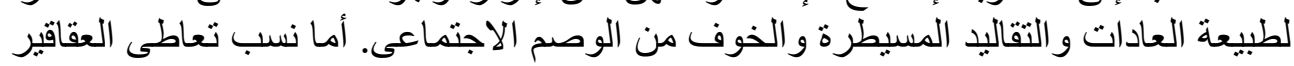

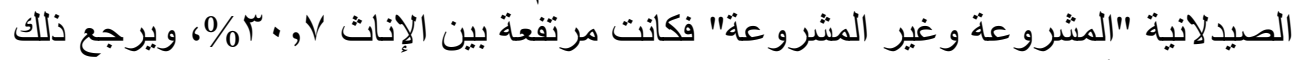

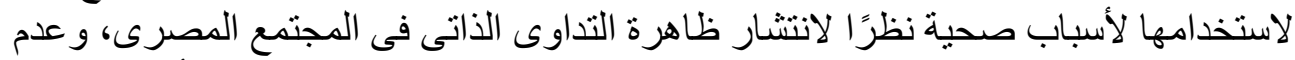

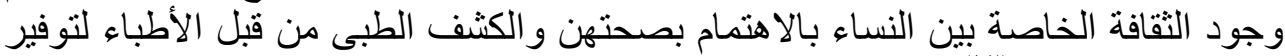

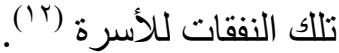

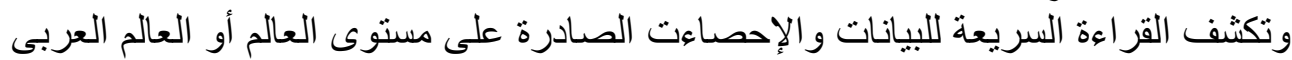

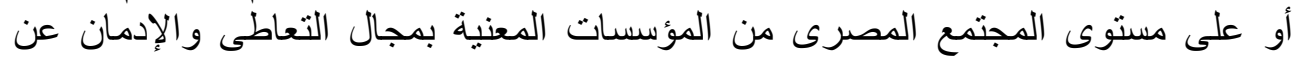

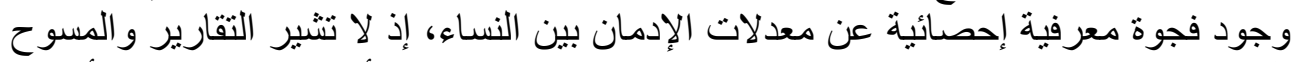

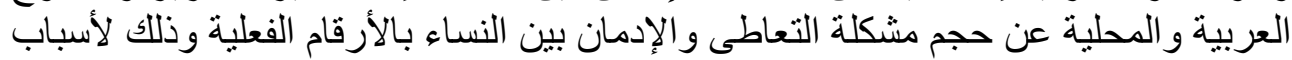

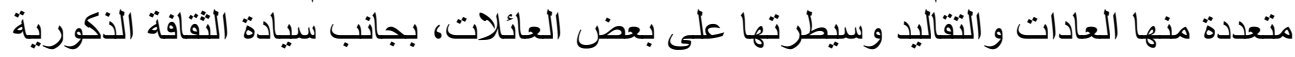

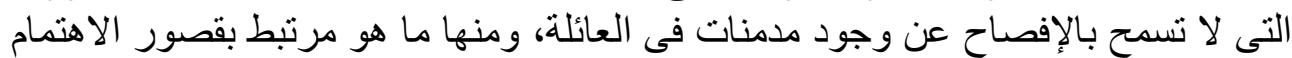

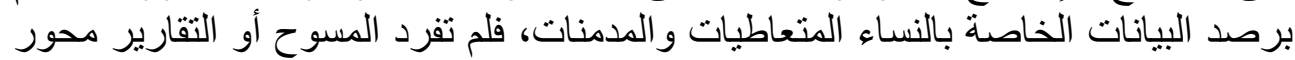

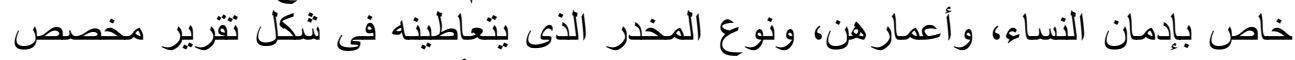

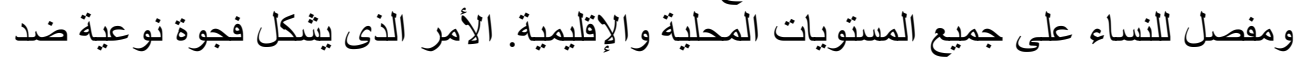
النساء.

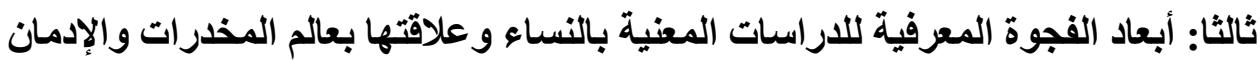

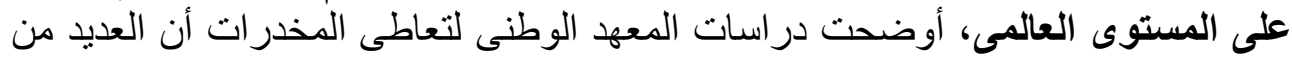

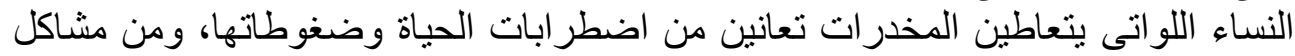

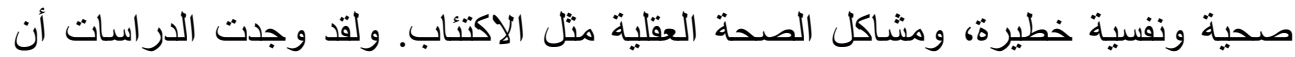

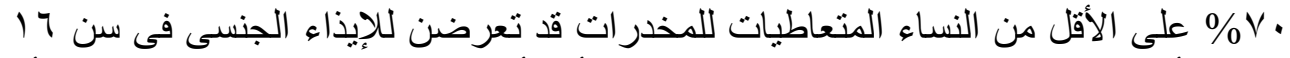

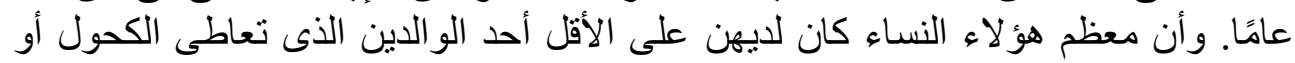

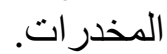
فى كثير من الأحيان، تعانى النساء اللواتى يتعاطين المخدر ات من تدنى احتر ام الذات وقات وقليل

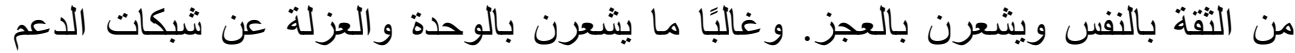

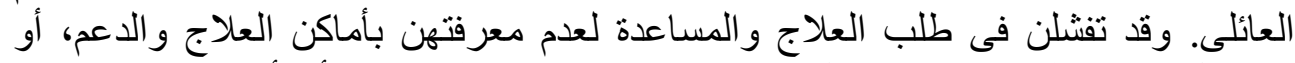

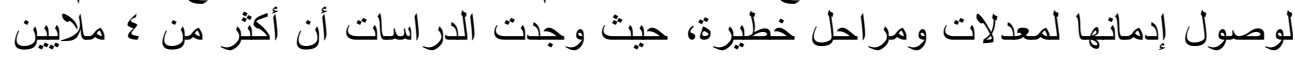

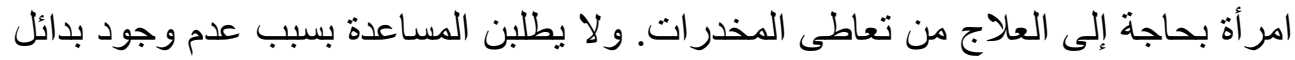




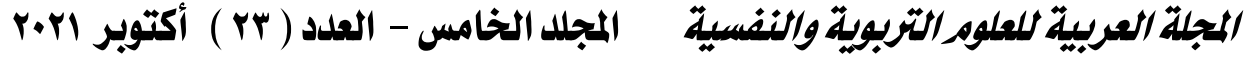

لرعاية أطفالهن، أو تخشى من أن السلطات قد تأخذ أطفالهن، وقد تخشى بعض النساء أن أن أنساء

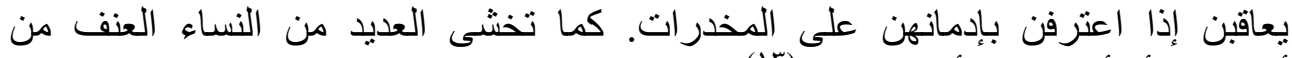

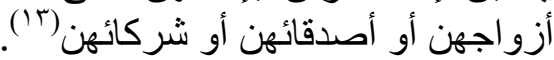

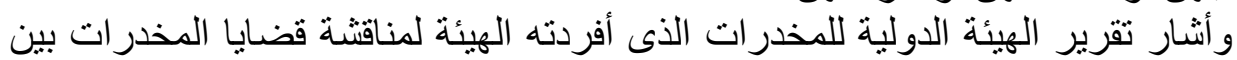

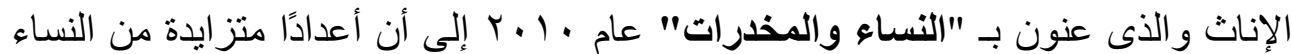

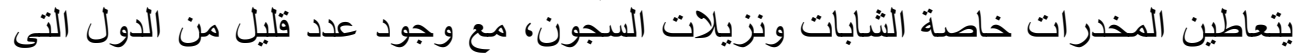

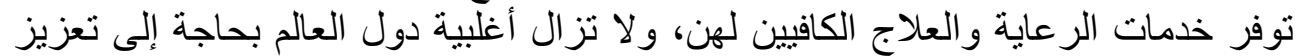

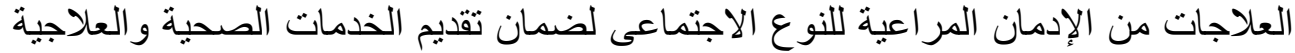

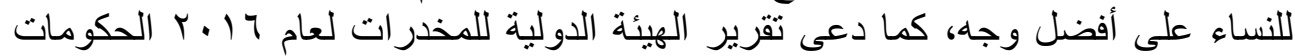
لبذل المزيد من الجهد لأخذ النساء بعين الاعتبار في السياسات و البرامج المئر المعنية بالمخدرات

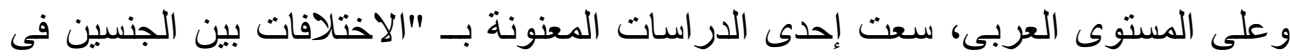

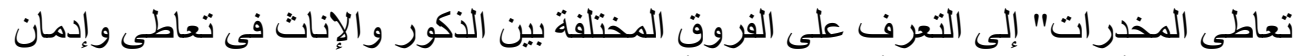

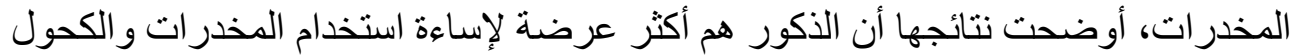

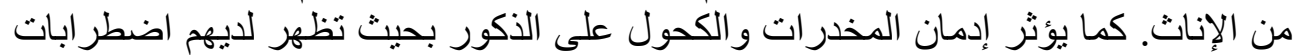

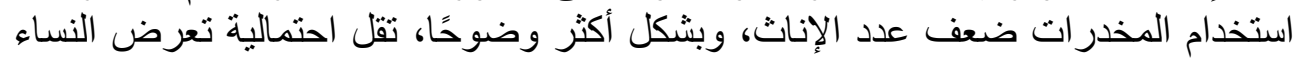

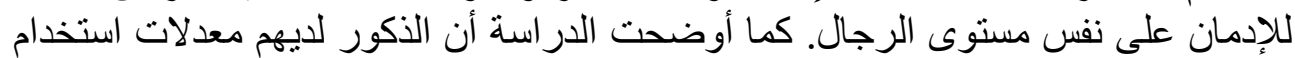

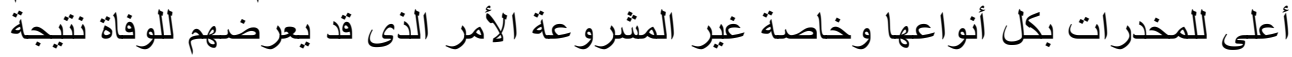

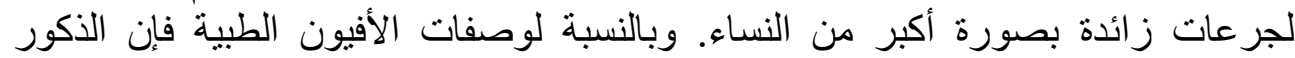

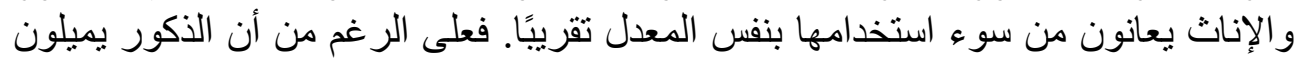

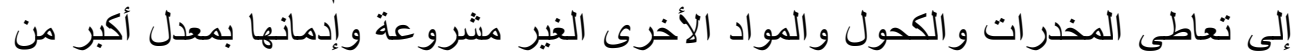

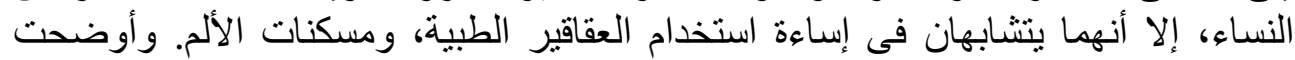

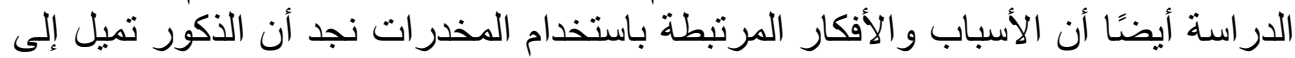

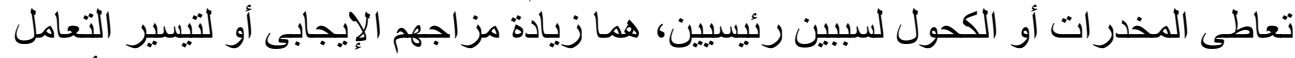

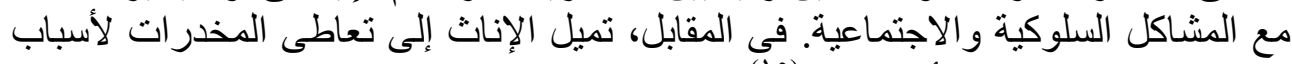
ذاتية تتعلق بقضايا نفسية أو عاطفية الأنية (10).

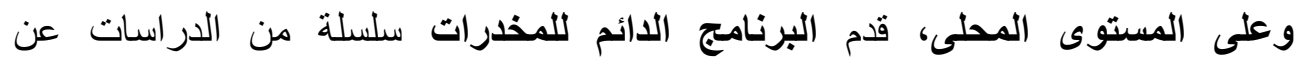

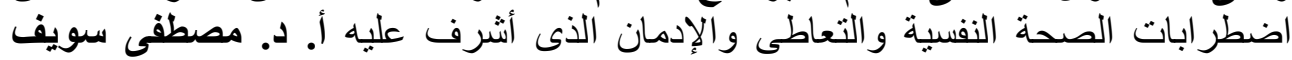

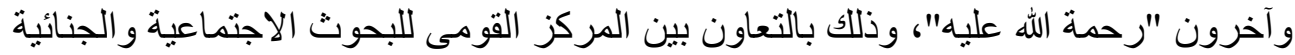

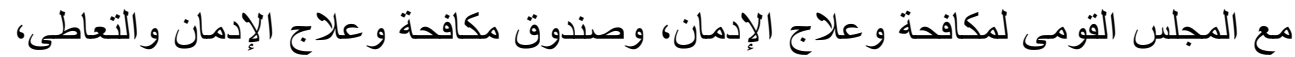

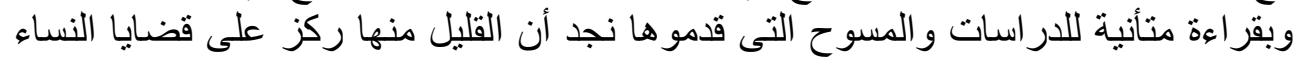

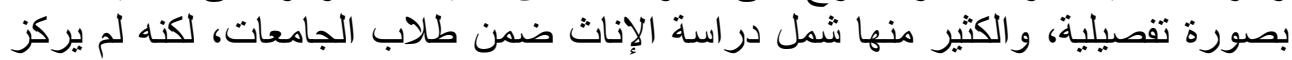




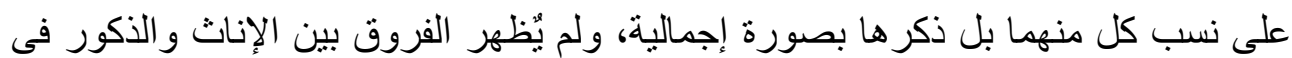

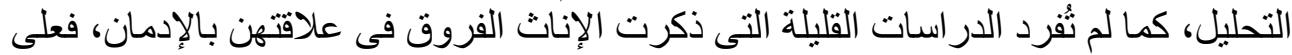

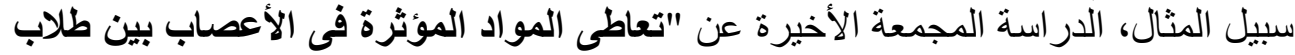

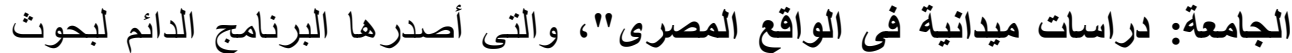

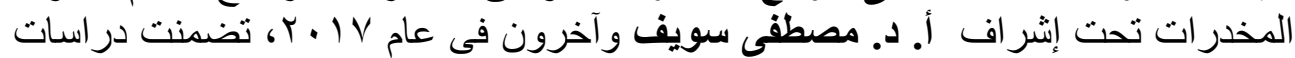

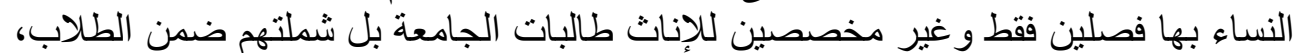

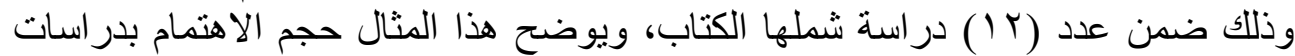

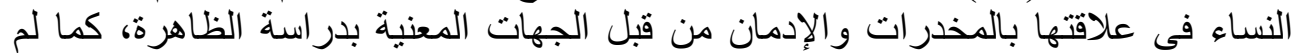

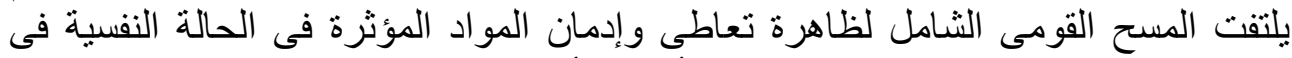

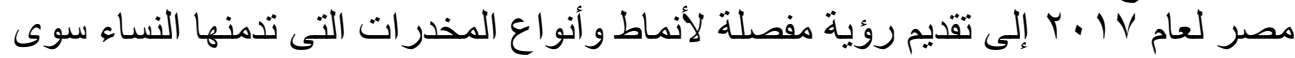

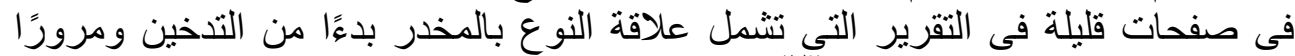

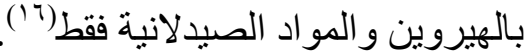

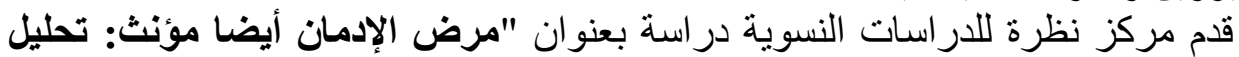

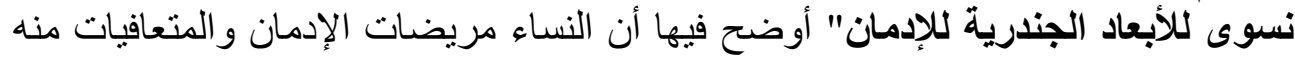

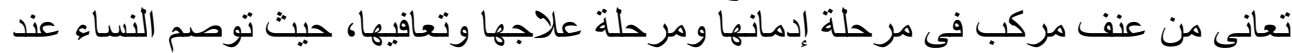

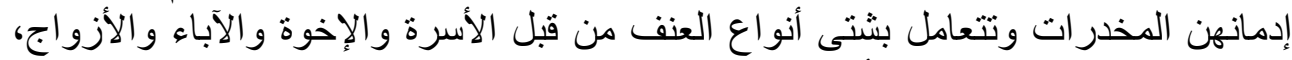

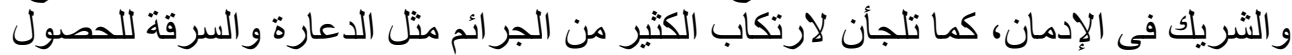

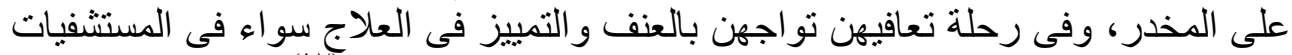

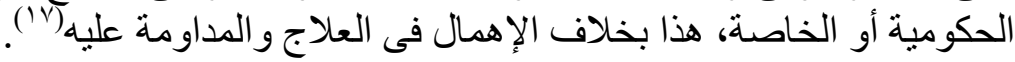

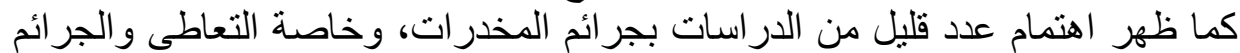

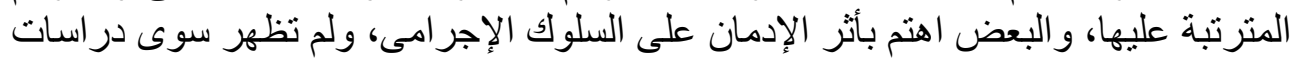

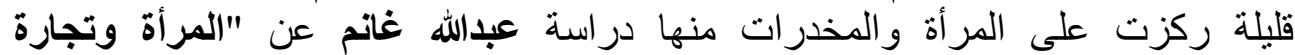

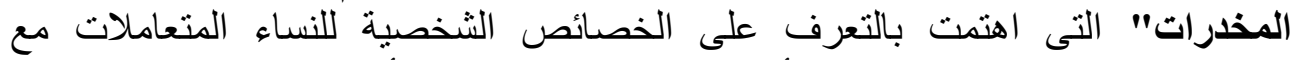

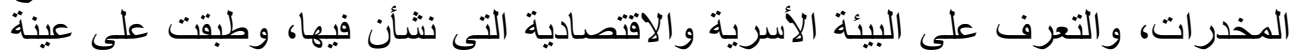

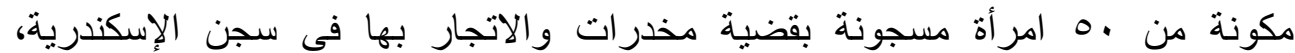

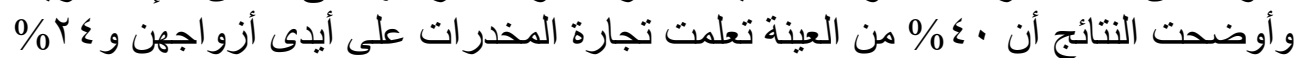

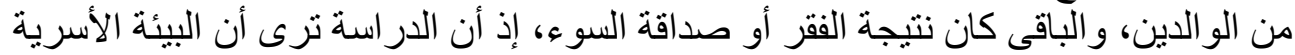

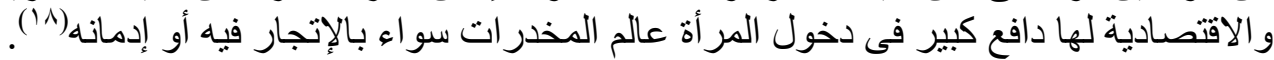

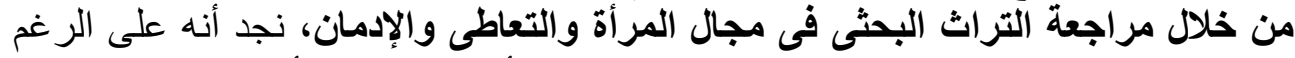

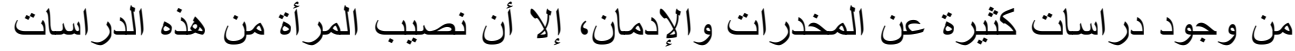

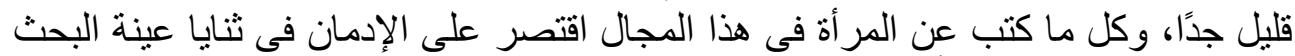

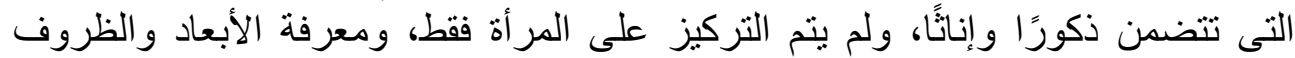




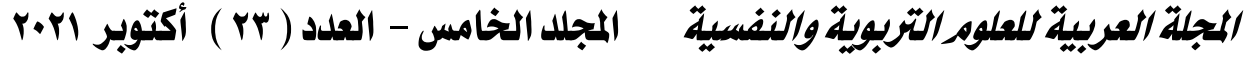

المختلفة التى دفعتها إلى الإدمان وخصوصية المر أة فى تجربة التعاطى والإدمان، و الجر ائم

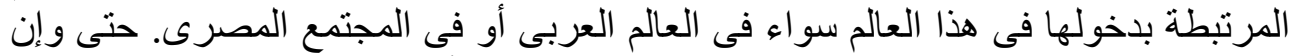

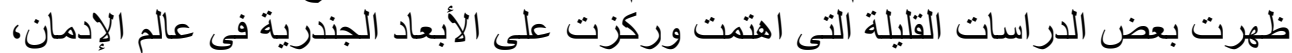

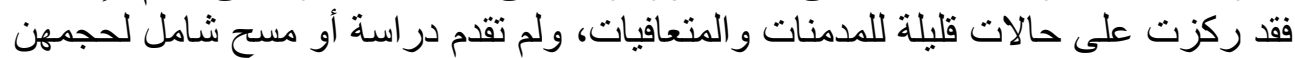

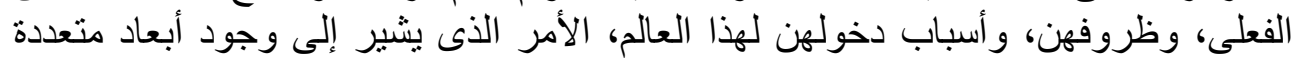

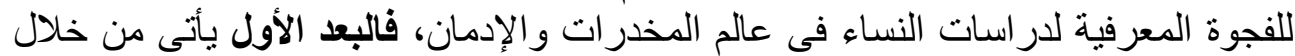

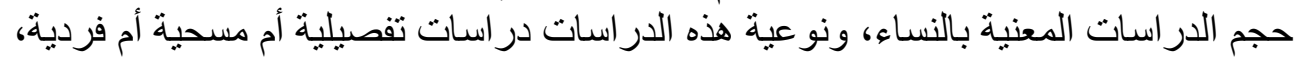

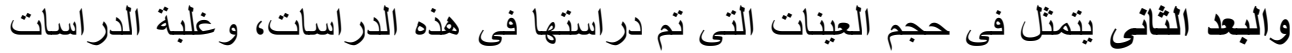

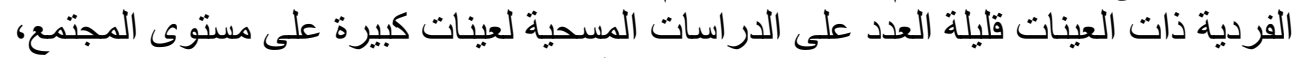

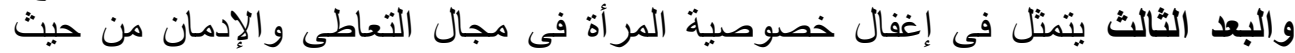

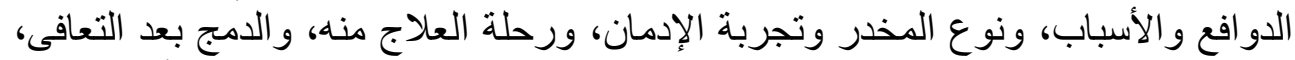

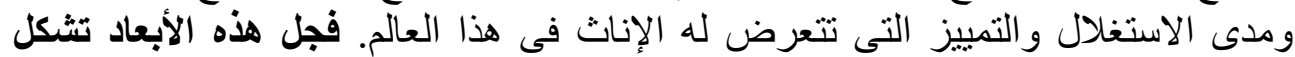

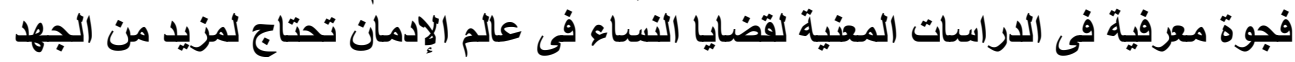

رابعا: أهم الأسباب والدئه الدوافع المختلفة لاخول النساء في عالم المخدرات والإدمان

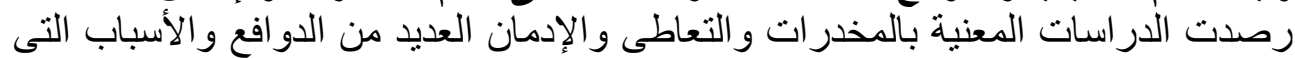

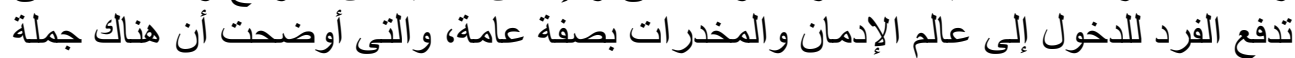

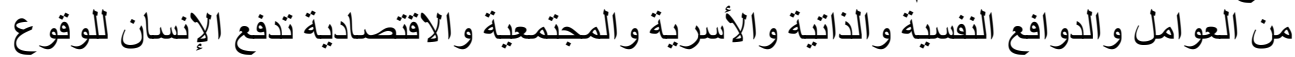

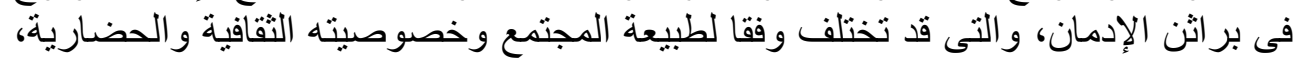

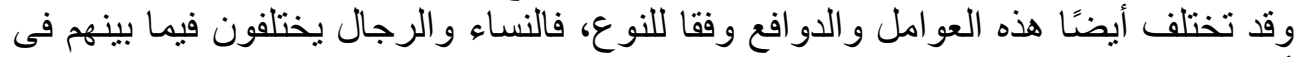

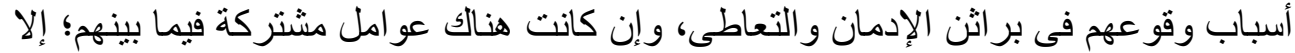

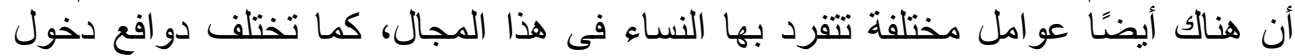

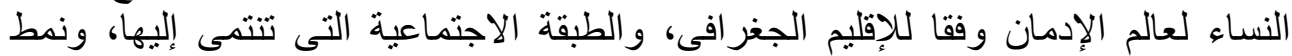

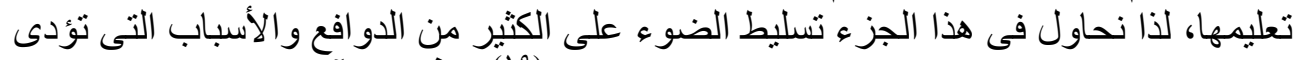

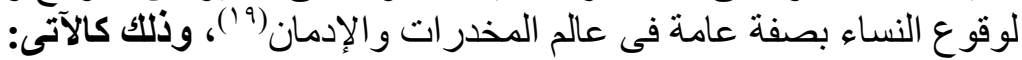

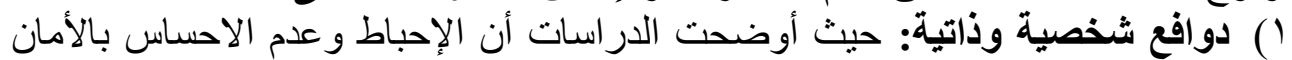

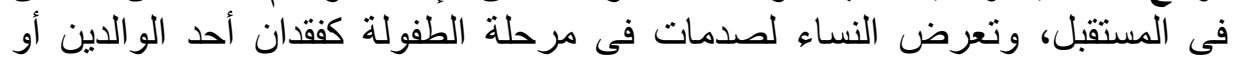

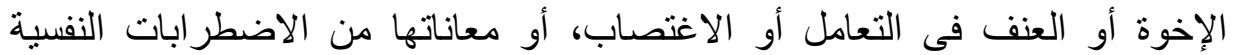

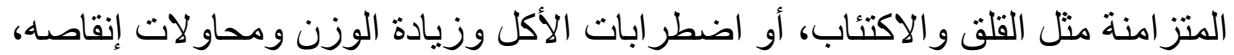

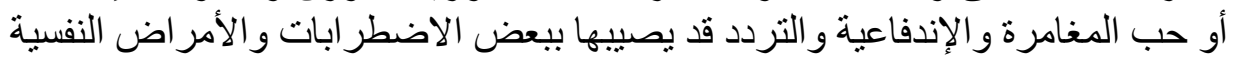
مما قد يجعلها فريسة سهلة لإخول والدول عالم الإدمان. 


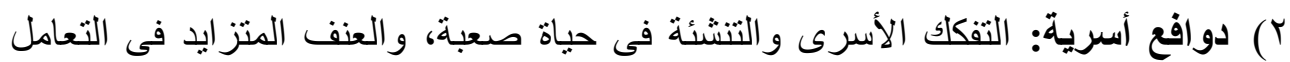

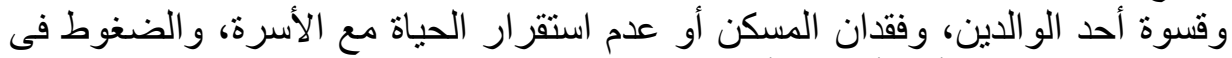

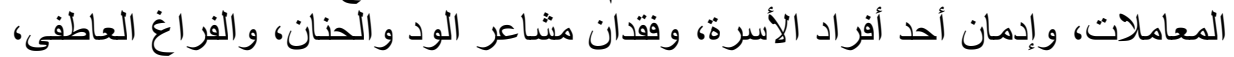

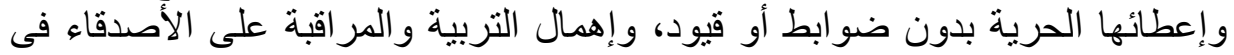

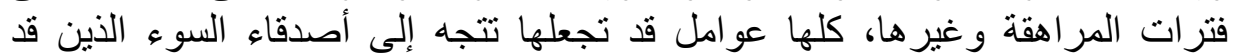
يدفعونها إلى التعاطى و الإدمان.

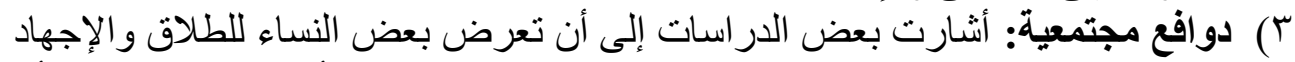

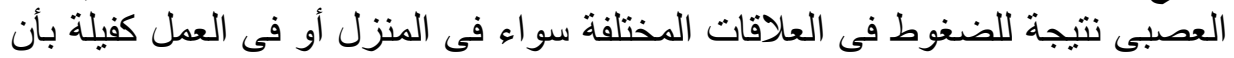

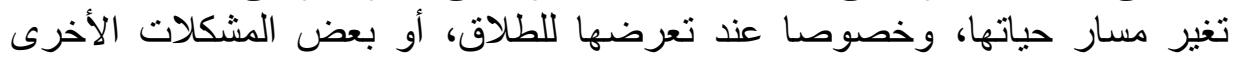

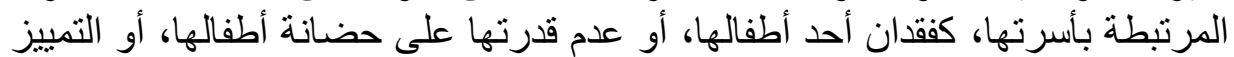

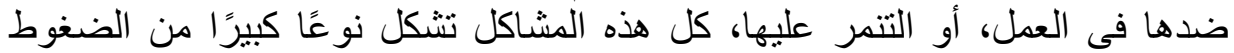

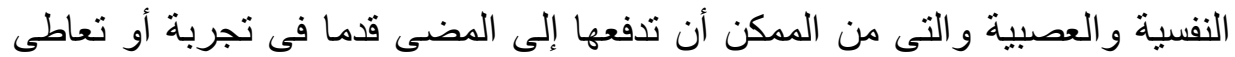

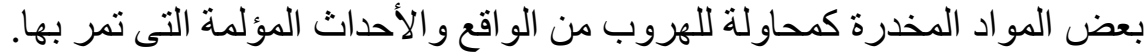

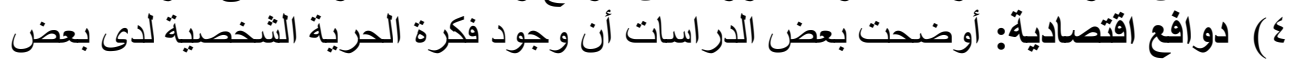

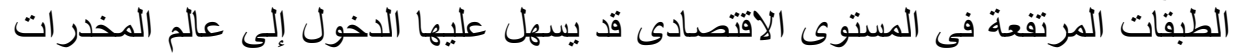

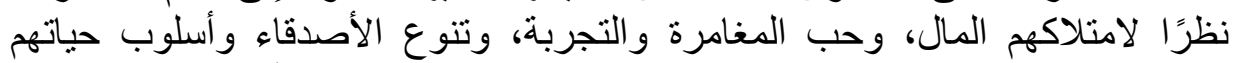

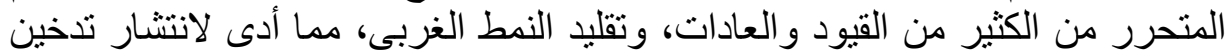

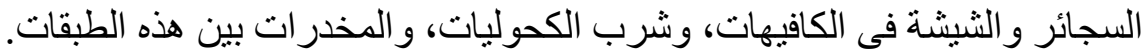

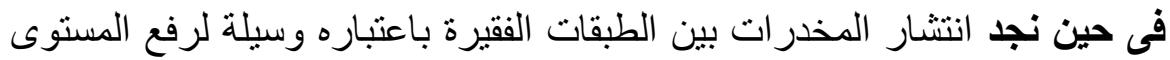

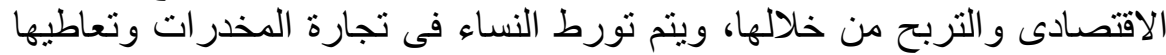

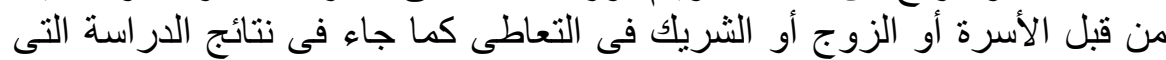

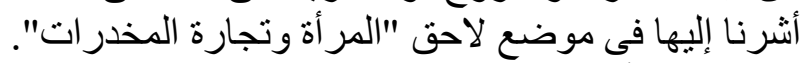

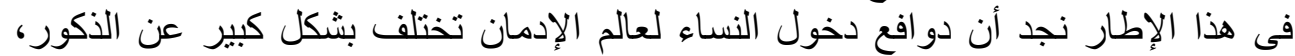

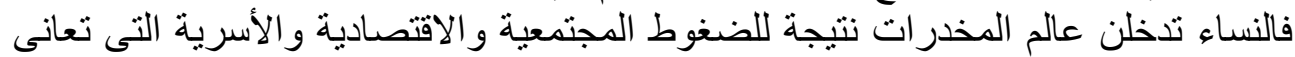

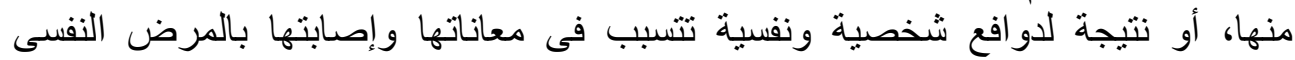

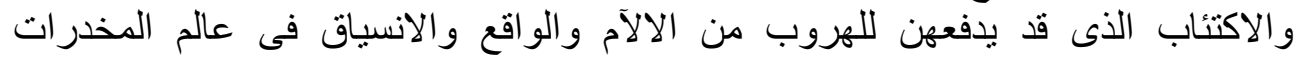

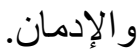

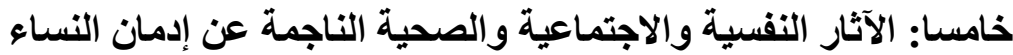

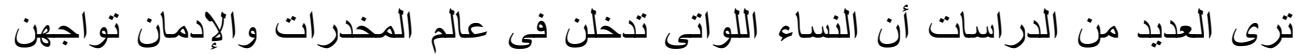

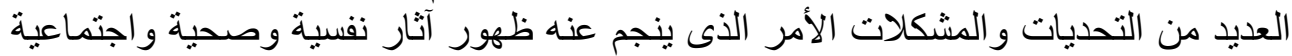

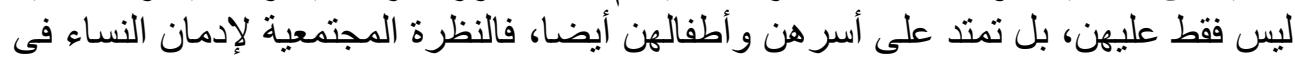

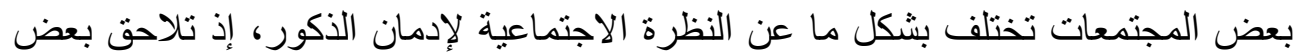




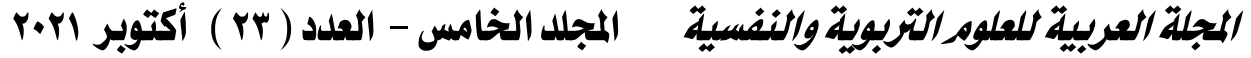

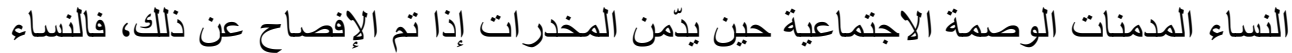

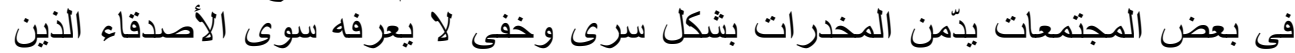

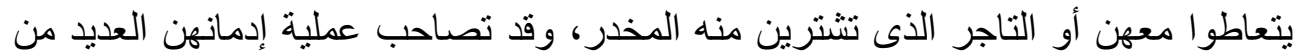

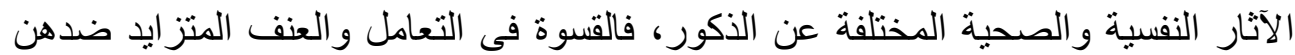

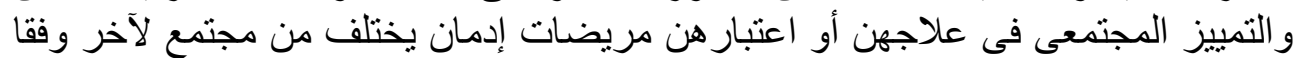

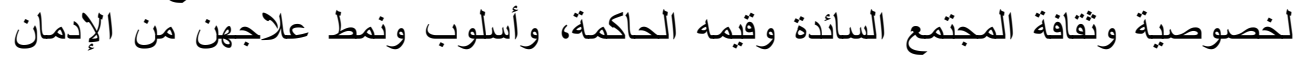

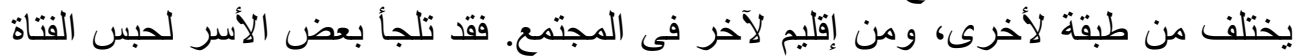

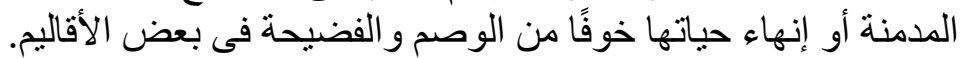
وفى هذا السياق يمكننا رصد أهم الأضر ار التى تلحق باءلى النساء المدمنات، وذللك على النحو

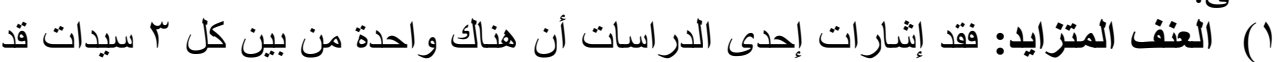

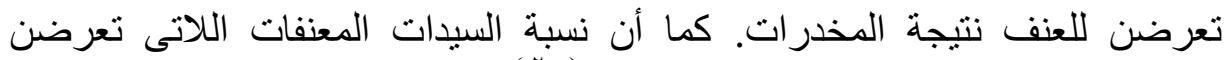

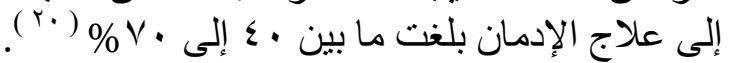

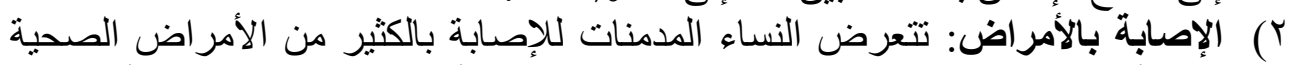

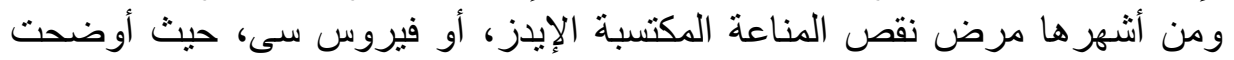

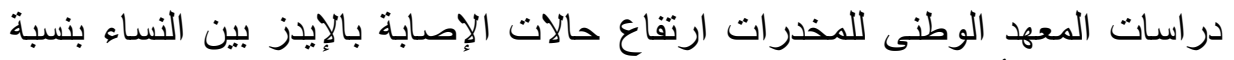

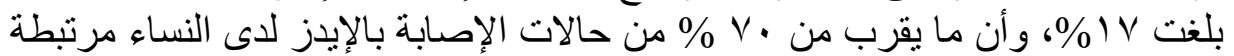

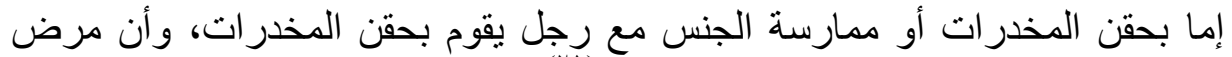

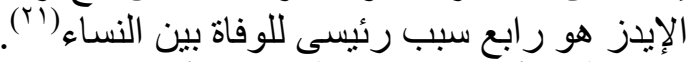

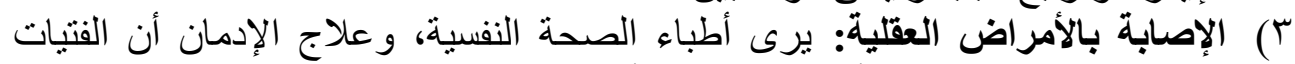

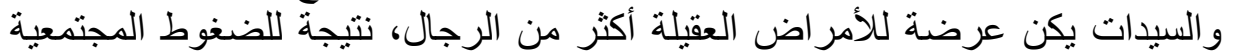

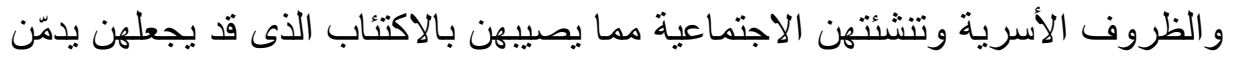

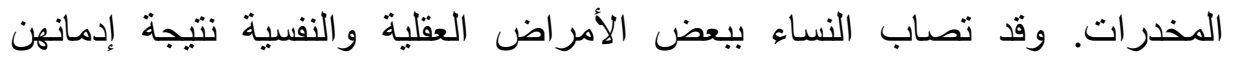

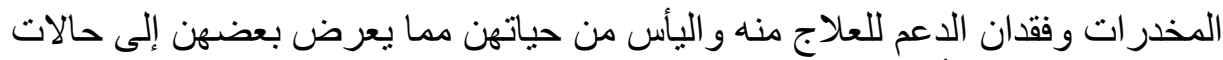

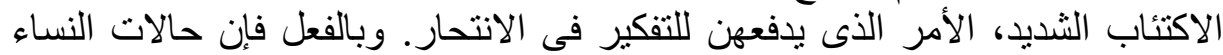

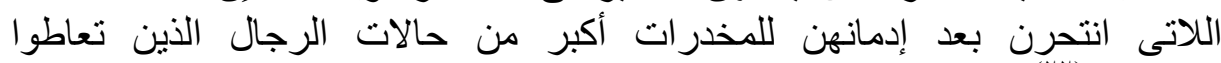

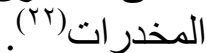
التثوهات الخلقية للجنين: ظهرت علاقة وطيدة بين حالات الحمل غير المرغوبة،

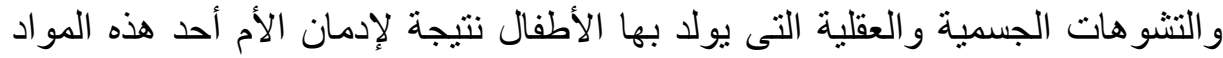

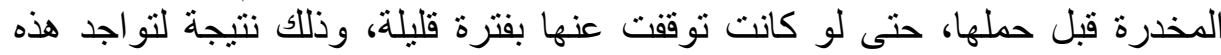

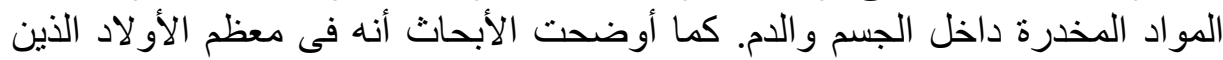

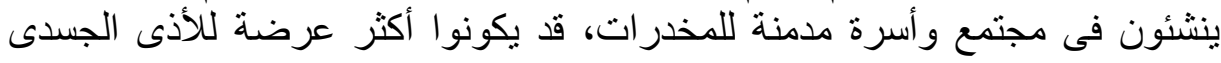


و النفسى، ومن المتوقع بنسبة كبيرة إدمان هؤلاء الأطفال المخدرات، عند وصولهم إلى

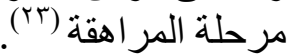

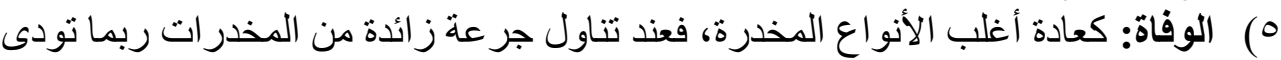

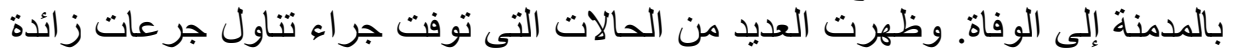

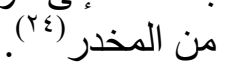

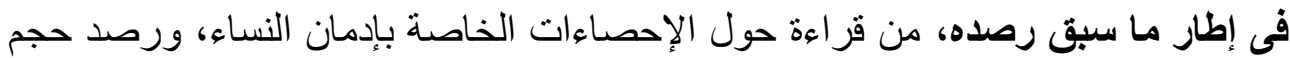

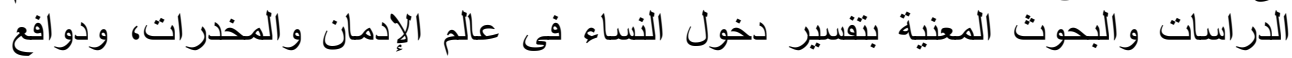

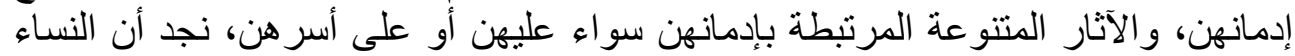

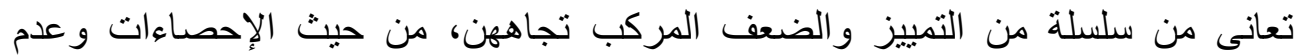

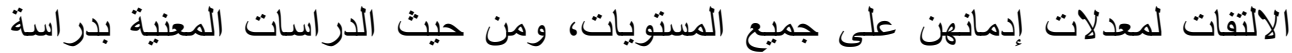

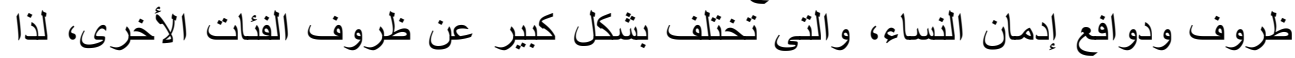

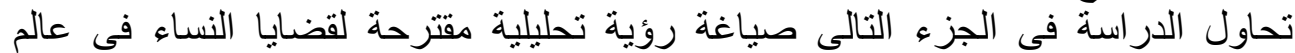

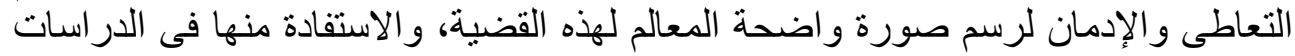

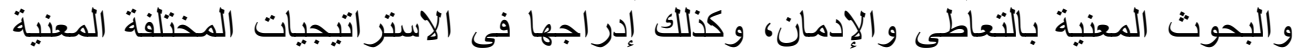
بظاهرة الإدمان. خاتمة: رؤية تحليلية سيكوسوسيولوجية مقترحة لرسم صورة النساء فى عالم التعاطى

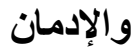
تبذل الدولة المصرية الكثثير من الجهود لمكافحة التعاطى والإدمان، وذللك من خلال إنشاء

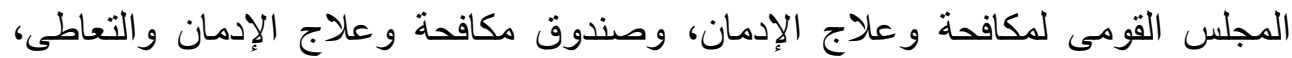

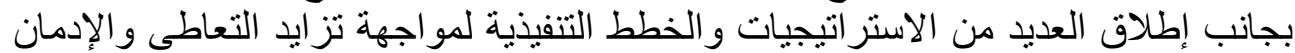

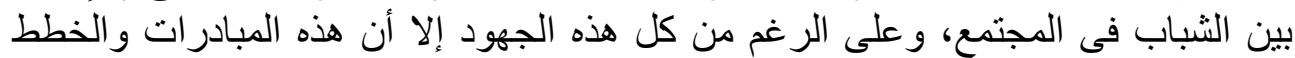

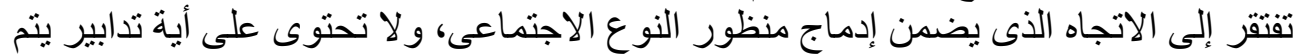

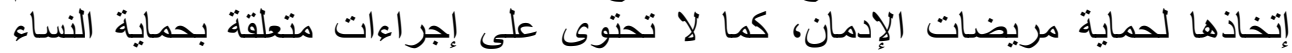

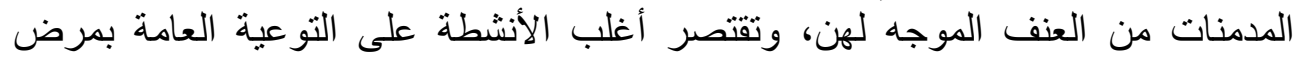

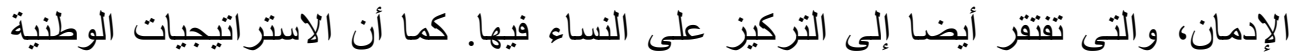

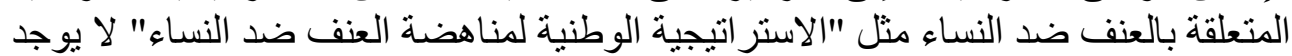

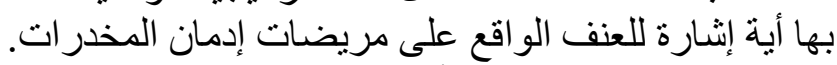

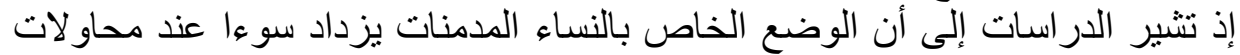

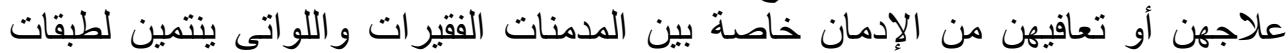

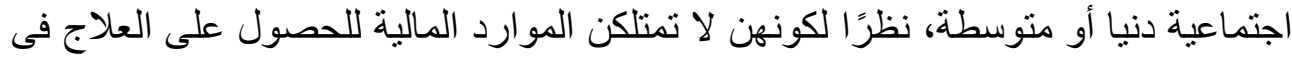

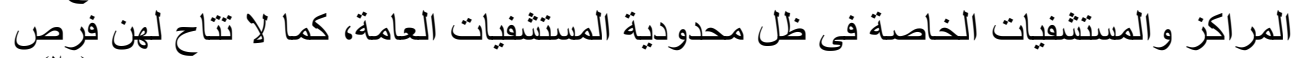
الحصول على الدعم و المساندة من عائلاتهن للوصول إلى بلى بداية الطريق لمرحلة التعافى (ب0). 


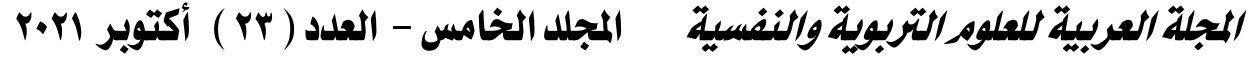

وفى هذا السياق، تحاول الدراسة صياغة رؤية تحليلية سيكوسوسيولوجية لقضايا النساء

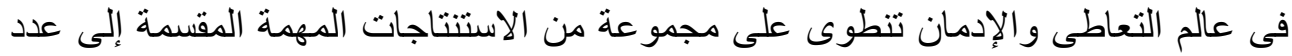

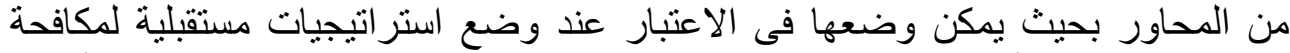

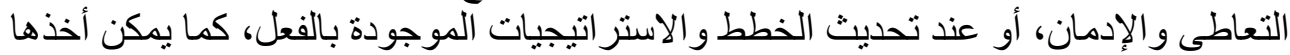

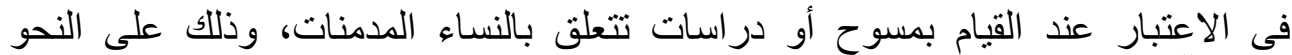

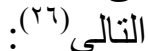

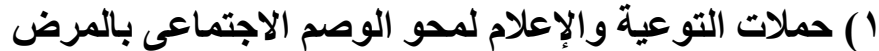

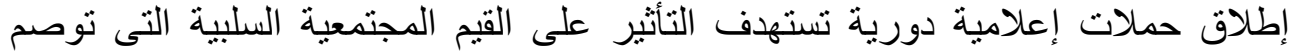

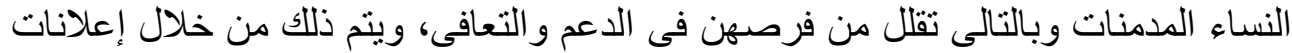

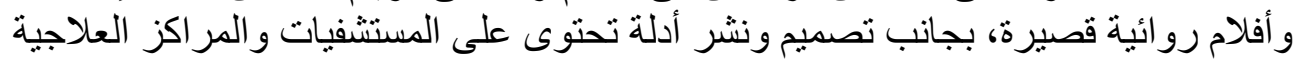

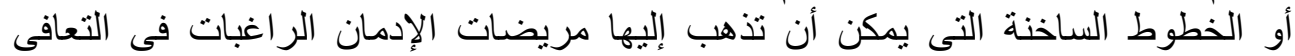

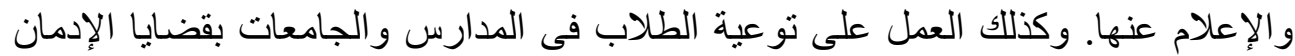

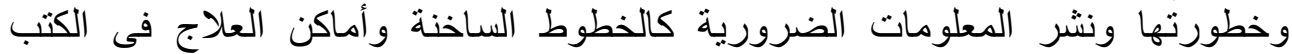
و المناهج التعليمية.

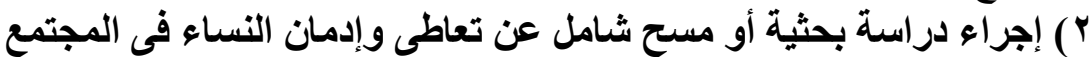

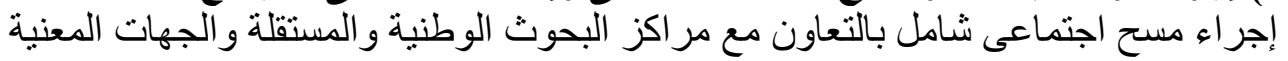

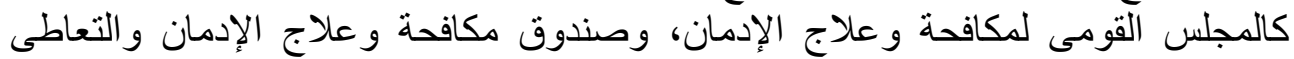

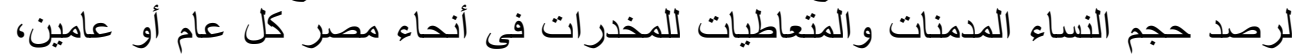

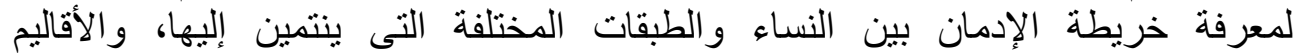
و المحافظات المختلفة التى يكثرن بها، وذللك ليتم تحديد الاحتباجات و التدابير العلاجية ولاية

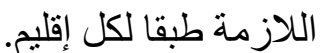

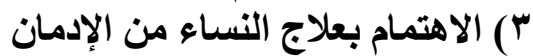
العمل على تخصيص الامنيص أقسام لعلاج المدمنات من الإمان النساء في المستشفيات و المر اكز العلاجية

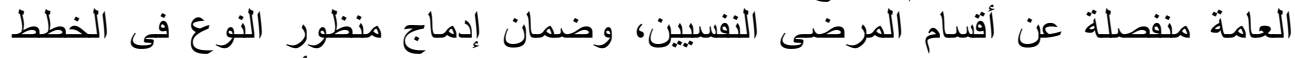

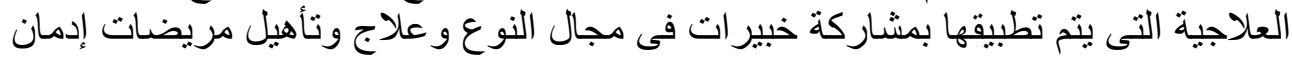

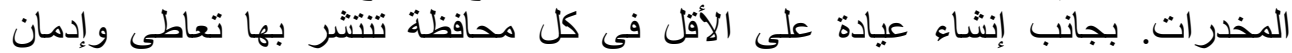

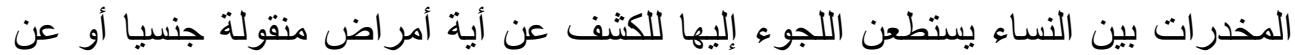

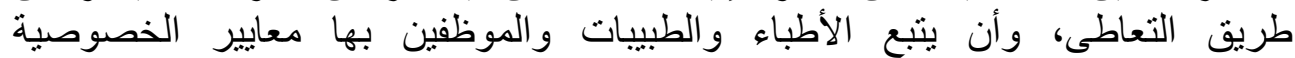

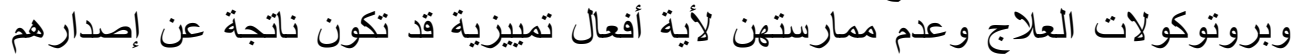

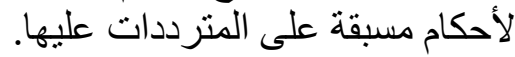
؛ ) الرعاية اللاحقة للمدمنات ودمجهن فئن فلى المجتمع 
العمل على إنشاء بيوت آمنة للمدمنات لكى يستطعن الإقامة المؤقتة فيها لتخطى المرحلة

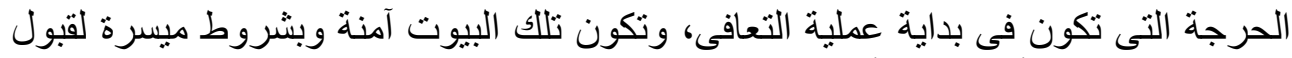

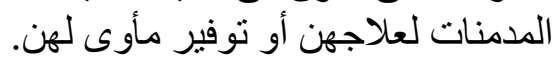

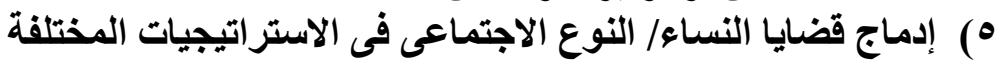

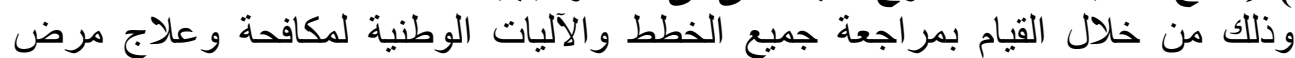

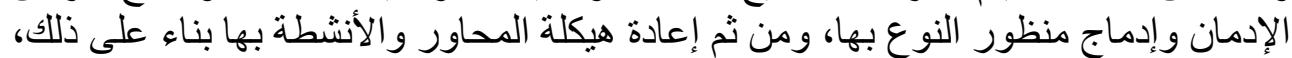

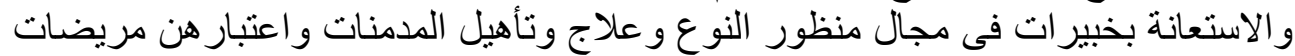

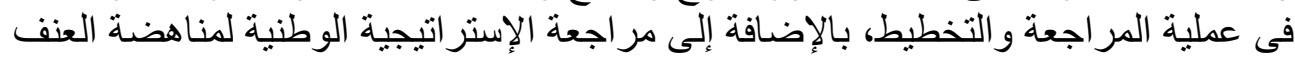

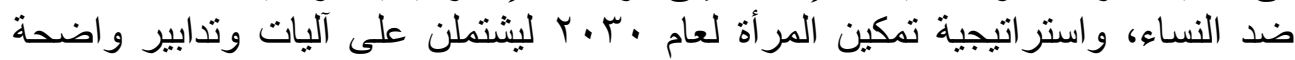

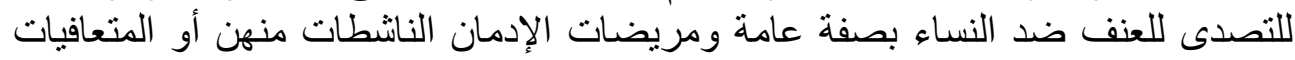
بصفة خاصة.

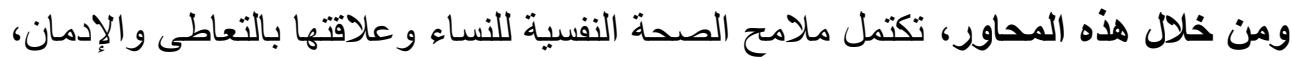

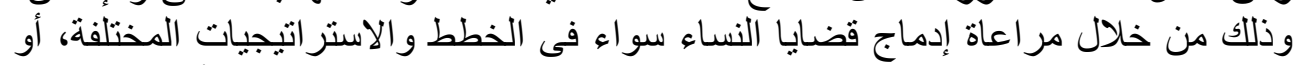

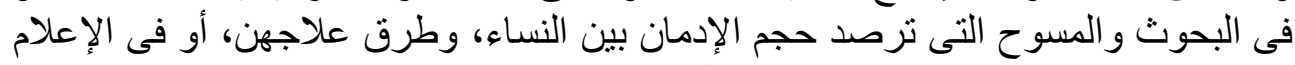

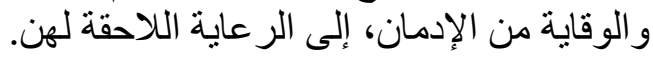




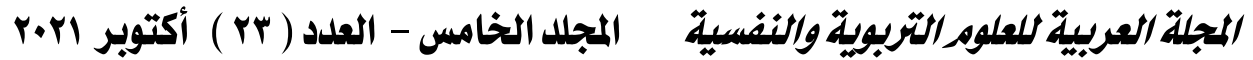

المراجع 1. منظمة الصحة العالمية، الصحة النفسية: حقائق و أنواع عن الأمراض النفسية، جنيف، $r \cdot r$.

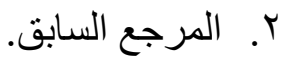
r. . محمد عبد الفتاح المهذى، الصحة النفسية للمر أة: قصص و اقعية من العيادة النفسية، دار

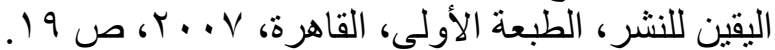

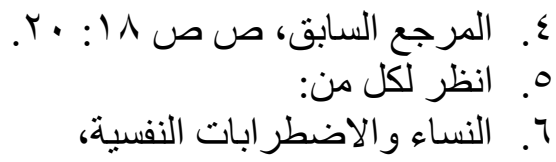

7. https://www.hopeeg.com/blog/show/women-mental-disorder

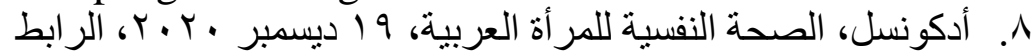
HTTPS://ADDCOUNSEL.COM/AR/9/ . 9

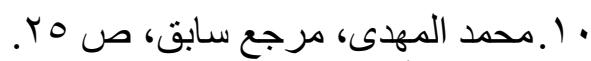

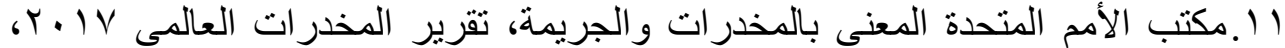

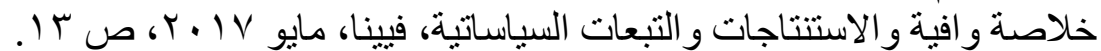

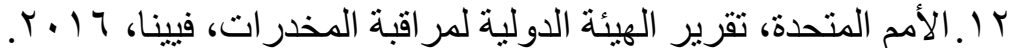
https://news.un.org/ar/story/2017/03/272062 . . 1 ع ا ـ مكتب الأمم المتحدة المعنى بالمخدرات والجريمة، تقرير المخدرات العالمى، فيينا، https://egypt.un.org/ar/

$$
\text { انظر لكل من: }
$$

10.أسباب انتشار المخدرات فى الوطن العربى، القاهرة، 10 ـ ب، انظر الرابط: https://www.afrigatenews.net/article

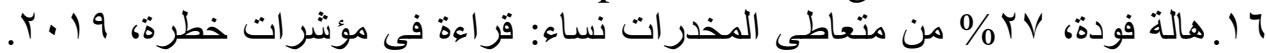

HTTPS://MARSAD.ECSSTUDIES.COM/6826 صندوق مكافحة وعلاج الإدمان والتعاطى، قراءة فى مؤشرات

6r.19

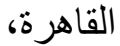

خطرة،

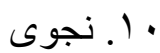

HTTPS://MARSAD.ECSSTUDIES.COM/6826

خليل وآخرون، المسح القومى الثامل لظاهرة تعاطى و إدمان المواد المؤثرة فى الحالة

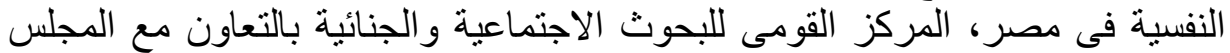

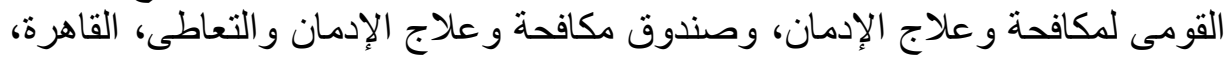

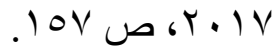


17. National Institute on Drugs Abuse, Women and Drug Abuse, 2020 https://archives.drugabuse.gov/publications/women-drugabuse/women-can-have-special-risk-factors-drug-abuse

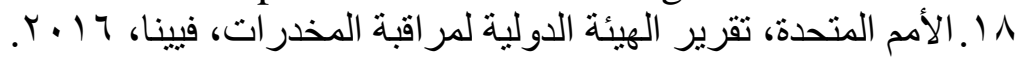
https://news.un.org/ar/story/2017/03/272062

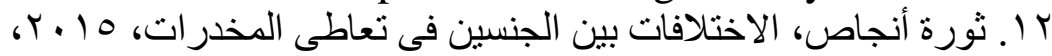

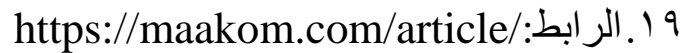

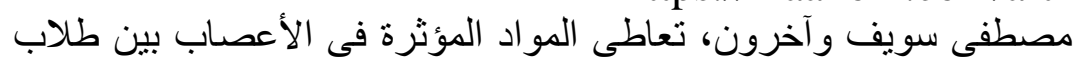

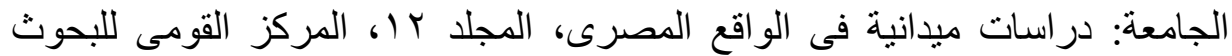

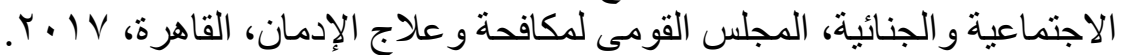

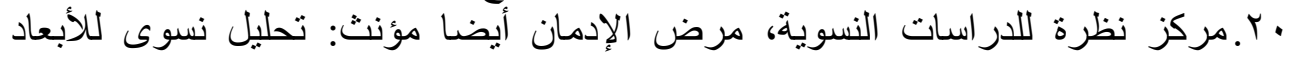

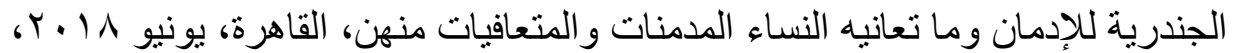

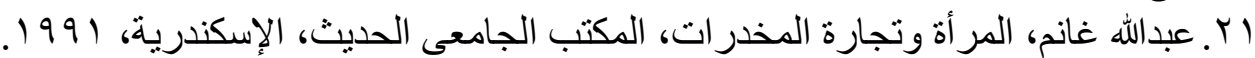

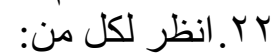

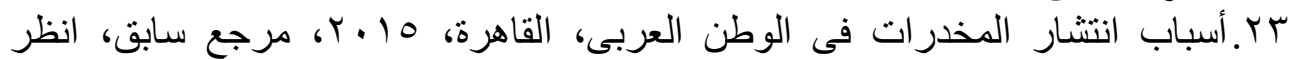

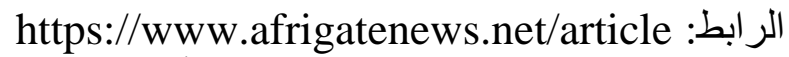

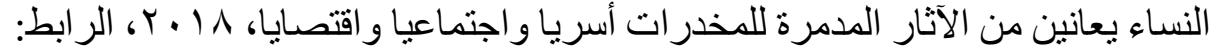

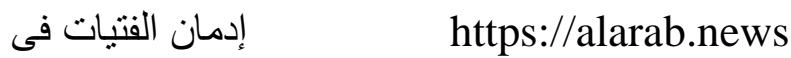

الدول العربية

https://www.hopeeg.com/blog/show/Drugs-and-girls

25. National Institute on Drugs Abuse, Women and Drug Abuse, 2020 https://archives.drugabuse.gov/publications/women-drugabuse/women-can-have-special-risk-factors-drug-abuse

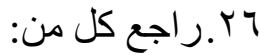
VV. النساء يعانين من الآثار المدمرة للمخدرات أسريا واجتماعيا واقتصايا، مرجع سابق، الر ابط: https://alarab.news

28. National Institute on Drugs Abuse, Women and Drug Abuse, 2020 https://archives.drugabuse.gov/publications/women-drugabuse/women-can-have-special-risk-factors-drug-abuse 


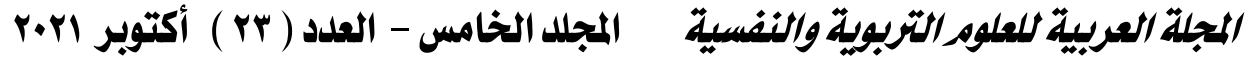

29. National Institute on Drugs Abuse, Women and Drug Abuse, 2020 https://archives.drugabuse.gov/publications/women-drugabuse/women-can-have-special-risk-factors-drug-abuse • •.روان مسعد ودينا عبد الخالق، فتيات دخلن عالم الدخدرات فى سن الطفولة، أغسطس

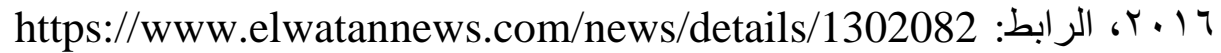

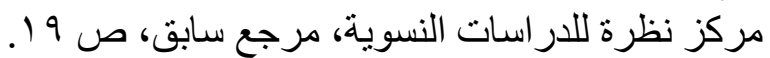

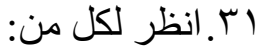

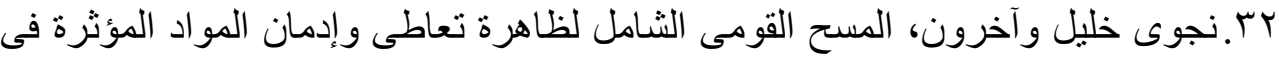

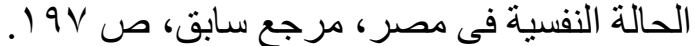

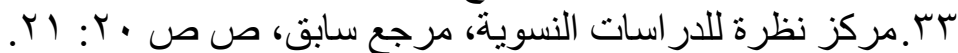

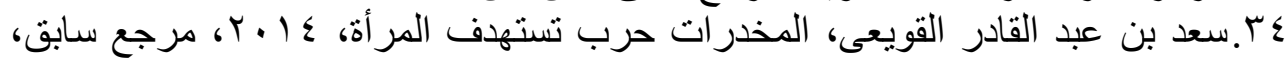

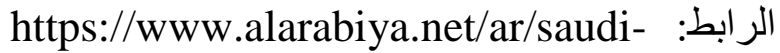
today/2014/11/15 
